\title{
Structure of spanning trees on the two-dimensional Sierpinski gasket
}

\author{
Shu-Chiuan Chang ${ }^{1}$ and Lung-Chi Chen ${ }^{2}$ \\ ${ }^{1}$ Department of Physics, National Cheng Kung University, Tainan 70101, Taiwan \\ ${ }^{2}$ Department of Mathematics, Fu Jen Catholic University, Taipei 24205, Taiwan
}

received $5^{\text {th }}$ February 2009, revised $21^{\text {st }}$ June 2010, $24^{\text {th }}$ August 2010, accepted $10^{\text {th }}$ September 2010.

Consider spanning trees on the two-dimensional Sierpinski gasket $S G(n)$ where stage $n$ is a non-negative integer. For any given vertex $x$ of $S G(n)$, we derive rigorously the probability distribution of the degree $j \in\{1,2,3,4\}$ at the vertex and its value in the infinite $n$ limit. Adding up such probabilities of all the vertices divided by the number of vertices, we obtain the average probability distribution of the degree $j$. The corresponding limiting distribution $\phi_{j}$ gives the average probability that a vertex is connected by 1,2, 3 or 4 bond(s) among all the spanning tree configurations. They are rational numbers given as $\phi_{1}=10957 / 40464, \phi_{2}=6626035 / 13636368, \phi_{3}=2943139 / 13636368$, $\phi_{4}=124895 / 4545456$.

Keywords: Spanning trees; Sierpinski gasket; exact solutions; limiting distribution; fractal geometry

\section{Introduction}

The enumeration of the number of spanning trees $N_{S T}(G)$ on a graph $G$ was first considered by Kirchhoff in the analysis of electric circuits [Kirchhoff(1847)] . It is a problem of fundamental interest in mathematics [Biggs(1993), Burton and Pemantle(1993), Lyons(2005), Welsh(1993)] and physics [Temperley(1972) $\mathrm{Wu}(1977)]$. The number of spanning trees corresponds to a special $q \rightarrow 0$ limit of the partition function of the $q$-state Potts model in statistical mechanics [Fortuin and Kasteleyn(1972), Wu(1982)], which in turn is related to the sandpile model [Cori and Borgne(2003), Dhar(2006)]. Just like other limits of the $q$-state Potts model, the spanning tree problem has been investigated intensely for decades, and has various applications in many areas. See, for example, [Wu and $\mathrm{Chao}(2004)]$ and references therein. It is also well known that there is a bijection between close-packed dimer coverings with spanning tree configurations on two related lattices [Temperley(1974)]. Some studies on the enumeration of spanning trees and the calculation of their asymptotic growth constants on regular lattices were carried out in Refs. [Chang and Shrock(2006)], [Chang and Wang(2006)], [Chang(2009)], [Shrock and Wu(2000)], [Tzeng and $\mathrm{Wu}(2000)]$. Once the total number of spanning trees and its asymptotic growth constant is obtained, the next step is to understand the geometric structure of spanning trees. One interesting question is the probability distribution of the degree of a certain vertex among all the spanning trees [Aldous(1991)]. The geometric properties of spanning trees on $\mathbb{Z}^{d}$ lattices, especially the square lattice, had been considered in [Burton and Pemantle(1993), Manna et al.(1992)].

1365-8050 @ 2010 Discrete Mathematics and Theoretical Computer Science (DMTCS), Nancy, France 
Fractals are geometric structures of (generally non-integer) Hausdorff dimension realized by repeated construction of an elementary shape on progressively smaller length scales, see [Falconer(2003)] and [Mandelbrot(1982)]. A well-known example of a fractal is the Sierpinski gasket that has been extensively studied in several contexts: [Alexander(1983)], [Daerden and Vanderzande(1998)], [Dhar and Dhar(1997)], [Dhar(2005)], [Domany et al.(1983)], [Gefen et al.(1980)], [Gefen and Aharony(1981)],

[Gefen et al.(1983,1984)], [Guyer(1984)], [Hattori et al.(1990,1992)], [Kozak and Balakrishnan(2002)(a)], ["Kozak and Balakrishnan(2002)(b)], [Rammal and Toulouse(1982)], [Chang and Chen (2008)(a)], [Chang and Chen (2008)(b)], [Chang and Chen (2008)(c)], [Chang and Chen (2009)]. Recently, the authors derived rigorously the number of spanning trees on the Sierpinski gasket and conjectured the result for arbitrary dimension [Chang et al.(2007)]. It is of interest to consider the geometric structure of spanning trees on self-similar fractal lattices which have scaling invariance rather than translational invariance. Different from the lattices that have translational invariance, e.g. the square lattice with periodic boundary conditions, the probability distribution of the degree on Sierpinski gasket depends on the vertex location. Thereby, it is natural to investigate the average of the probability distribution of the degree over all the vertices on $S G(n)$ as $n$ tends to infinite, and compare the values with the corresponding results on the infinite square lattice which is also 4-regular. In this paper, we shall present the probability distribution of the degree at any given vertex $x$ on the two-dimensional Sierpinski gasket and the average over all the vertices on $S G(n)$, and the limiting distribution when the number of vertices goes to infinity.

\section{Preliminaries}

We first recall some relevant definitions for spanning trees and the Sierpinski gasket in this section. A connected graph (without loops) $G=(V, E)$ is defined by its vertex (site) and edge (bond) sets $V$ and $E$ [Biggs(1993), Harary(1969)]. Let $v(G)=|V|$ be the number of vertices and $e(G)=|E|$ the number of edges in $G$. A spanning subgraph $G^{\prime}$ is a subgraph of $G$ with the same vertex set $V$ and an edge set $E^{\prime} \subseteq E$. As a tree is a connected graph with no circuits, a spanning tree on $G$ is a spanning subgraph of $G$ that is a tree and hence $e\left(G^{\prime}\right)=v(G)-1$. The degree or coordination number $k_{i}$ of a vertex $v_{i} \in V$ is the number of edges attached to it. A $k$-regular graph is a graph with the property that each of its vertices has the same degree $k$. In general, one can associate an edge weight to each edge connecting adjacent vertices $v_{i}$ and $v_{j}$ (see, for example [Tzeng and $\left.\mathrm{Wu}(2000)\right]$ ). For simplicity, all edge weights are set to one throughout this paper, so that the weight of each spanning tree is the same.

The construction of the two-dimensional Sierpinski gasket $S G(n)$ at stage $n$ is shown in Fig. 1. At stage $n=0$, it is an equilateral triangle; while stage $n+1$ is obtained by the juxtaposition of three $n$-stage structures. For the two-dimensional Sierpinski gasket $S G(n)$, the numbers of edges and vertices are given by

$$
e(S G(n))=3^{n+1}, \quad v(S G(n))=\frac{3}{2}\left(3^{n}+1\right) .
$$

Except the three outmost vertices which have degree two, all other vertices of $S G(n)$ have degree four. In the large $n$ limit, $S G$ is 4-regular.

Let us define the notation for the vertices of $S G(n)$ to be used. An illustration for $S G(4)$ is shown in Fig. 2. The denotation of the vertices is given progressively with increasing number of digits in the subscript as follows. First of all, fix $o$ as the leftmost vertex. Consider the $S G(m)$ with $0 \leq m \leq n$ which has $o$ as its leftmost vertex, and denote $a_{m}$ and $b_{m}$ as its rightmost and topmost vertices, respectively. $c_{m}$ is defined such that the vertices $a_{m}, b_{m}$ and $c_{m}$ demarcate the largest lacunary triangle of $S G(m+1)$. We 
then define the vertex in the middle of the line connecting $a_{m}$ and $a_{m+1}$ with $m \geq 1$ as $a_{m, 1}$. Similarly, $a_{m, 1}$ and the associated $b_{m, 1}$ and $c_{m, 1}$ demarcate a lacunary triangle with $b_{m, 1}$ on the left and $c_{m, 1}$ on the right. Next for $m \geq 2$, we append the subscript $m, 1,0$ for the vertices of the largest lacunary inside the triangle with outmost vertices $a_{m}, a_{m, 1}, b_{m, 1}$; the subscript $m, 1,1$ for the vertices of the largest lacunary inside the triangle with outmost vertices $a_{m, 1}, a_{m+1}, c_{m, 1}$; the subscript $m, 1,2$ for the vertices of the largest lacunary inside the triangle with outmost vertices $b_{m, 1}, c_{m, 1}, c_{m}$, etc. In general for the vertices of $S G(n)$, we use the notation $x_{\vec{\gamma}}$ where $x=a, b, c$ and the subscript $\vec{\gamma}=\left(\gamma_{1}, \ldots, \gamma_{s}\right)$ has $s$ components with $1 \leq s \leq n, 1 \leq \gamma_{1}<n$ and $\gamma_{k} \in\{0,1,2\}$ for $k \in\{2,3, \ldots, s\}$. For the vertices above the extended line connecting $o$ and $c_{0}$, we will also use the notation $\tilde{x}_{\vec{\gamma}}$ such that it is the reflection of the vertex $x_{\vec{\gamma}}$ with respect to this line. For example, $a_{22}=\tilde{b}_{21}, b_{221}=\tilde{a}_{212}, c_{222}=\tilde{c}_{211}$, etc. The advantage of such a vertex notation is that the quantities to be studied for the vertices $x_{\gamma_{1}, \ldots, \gamma_{s}}$ with $s \geq 2$ components in the subscript can be expressed in terms of the quantities for the vertices with $s-1$ components in the subscript as shown in Section 5 .

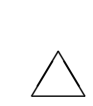

$S G(0) \quad S G(1)$

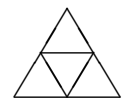

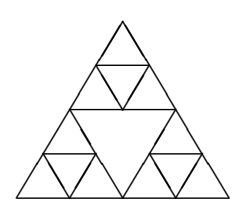

$S G(2)$

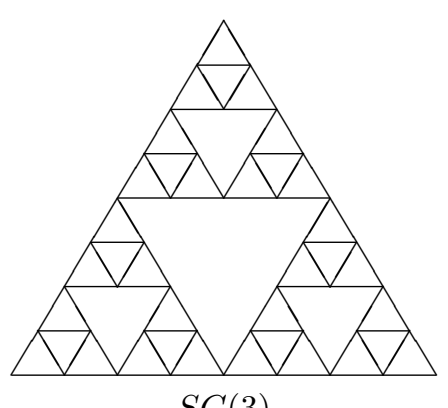

$S G(3)$

Fig. 1: The first four stages $n=0,1,2,3$ of the two-dimensional Sierpinski gasket $S G(n)$.

Let us define the following quantities as in [Chang et al.(2007)].

Definition 2.1 Consider the two-dimensional Sierpinski gasket $S G(n)$ at stage $n$. (i) Define $f(n) \equiv$ $N_{S T}(S G(n))$ as the number of spanning trees. (ii) Define $g(n)$ as the number of spanning subgraphs with two trees such that the vertex $b_{n}$ belongs to one tree and the set of vertices $\left\{o, a_{n}\right\}$ belongs to the other tree. (iii) Define $h(n)$ as the number of spanning subgraphs with three trees such that each of the outmost vertices $o, a_{n}$ and $b_{n}$ belongs to a different tree.

Notice that for the spanning subgraph configurations counted by $g(n)$, it is possible that the vertex $b_{n}$ is an isolated vertex with no bonds of trees connecting to it. A similar statement applies to the outmost vertices $o, a_{n}, b_{n}$ for the spanning subgraph configurations counted by $h(n)$. For a given vertex, we would like to investigate the number of bonds of spanning trees connecting to it among all the spanning tree configurations. We have the following definitions.

Definition 2.2 Consider the two-dimensional Sierpinski gasket $S G(n)$ at stage $n$. For a certain vertex $x \in V(S G(n))$, the number of bond(s) connecting to it in a spanning tree configuration is denoted as $j \in\{1,2,3,4\}$ or $i \in\{0,1,2,3,4\}$. (i) Define $f_{j}(n, x)$ as the number of spanning trees such that there is (are) $j$ bond(s) connecting the vertex $x$. Define the probability $F_{j}(n, x)=f_{j}(n, x) / f(n)$. (ii) Define $g_{i}(n, x)$ as the number of spanning subgraphs with two trees such that the vertex $b_{n}$ belongs to one tree 


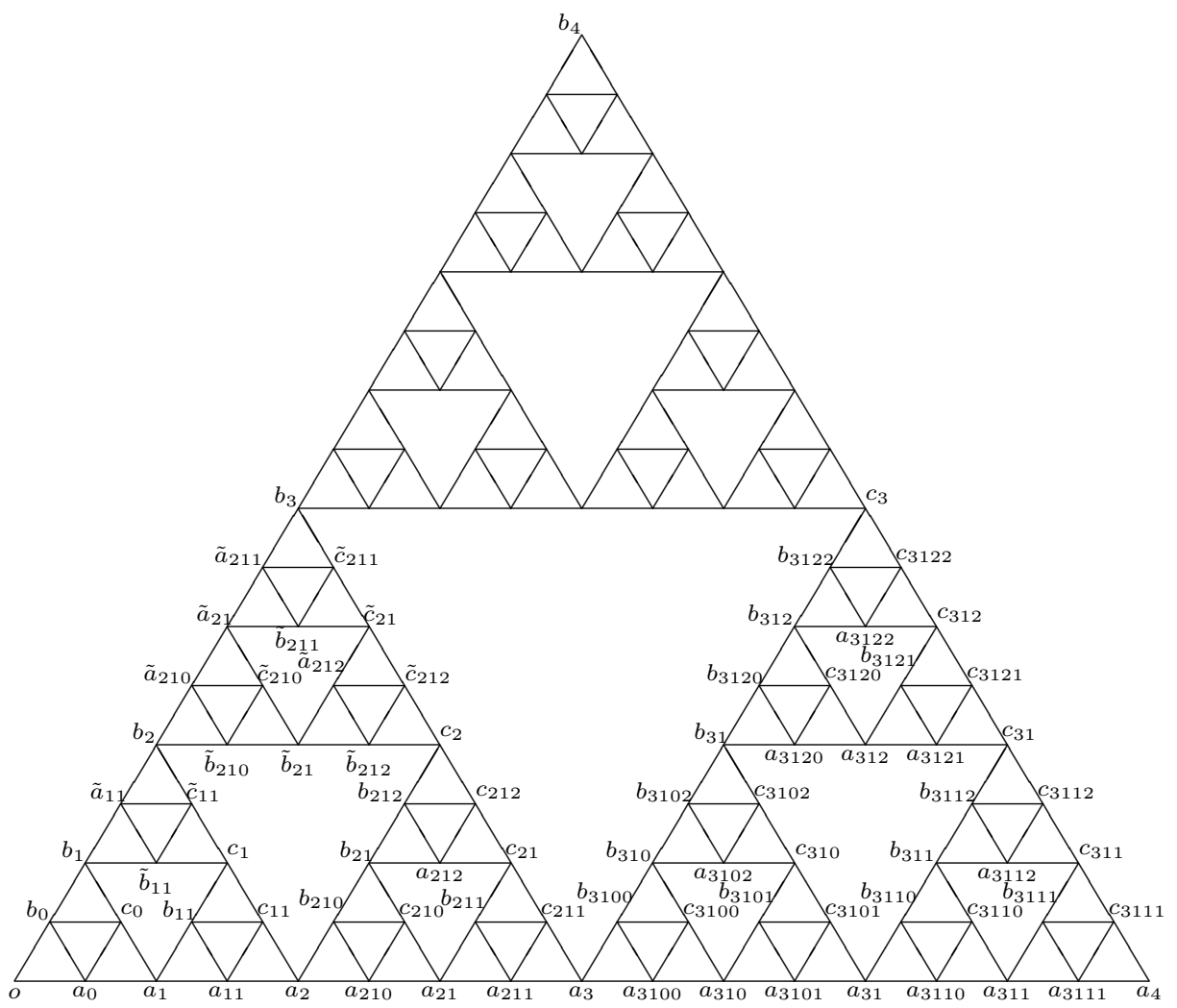

Fig. 2: The notation for the vertices of the Sierpinski gasket $S G(4)$. The vertices $\tilde{x}_{\vec{\gamma}}$ inside the triangle $\left(b_{3}, c_{3}, b_{4}\right)$ are reflections of the vertices $x_{\vec{\gamma}}$ inside the triangle $\left(a_{3}, c_{3}, a_{4}\right)$ with respect to the line connecting $o$ and $c_{3}$, and are not shown. 
and the set of vertices $\left\{o, a_{n}\right\}$ belongs to the other tree, and there is (are) $i$ bond $(s)$ connecting the vertex $x$. Define the probability $G_{i}(n, x)=g_{i}(n, x) / g(n)$. (iii) Define $h_{i}(n, x)$ as the number of spanning subgraphs with three trees such that each of the outmost vertices $o, a_{n}$, $b_{n}$ belongs to a different tree, and there is (are) $i$ bond $(s)$ connecting the vertex $x$. Define the probability $H_{i}(n, x)=h_{i}(n, x) / h(n)$.

For any vertex $x$ of $S G(n)$, the following relations for the probabilities should be satisfied,

$$
\sum_{j=1}^{4} F_{j}(n, x)=\sum_{j=0}^{4} G_{j}(n, x)=\sum_{j=0}^{4} H_{j}(n, x)=1,
$$

which serves as a check for the results obtained.

In this paper, we derive rigorously $F_{j}(n, x)$ or $f_{j}(n, x)$ for an arbitrary vertex $x \in V(S G(n))$ with $j=1,2,3,4$. Such probability on translational invariance lattices in the infinite-vertex limit is independent of the vertex location. In contrast, as the Sierpinski gasket is a self-similar fractal lattice which has scaling invariance rather than translational invariance, our results depend on the location of $x$. We shall consider the simplest vertex $x=o$ to obtain $F_{j}(n, o)$ as Theorem 3.1 and its infinite $n$ limit as Corollary 3.1 in Section 3 then move on to the vertices $x \in\left\{a_{m}, b_{m}, c_{m}\right\}$ with $0 \leq m<n$ to have Theorem 4.1 and Corollary 4.1 in Section $4 . F_{j}(n, x)$ for the remaining vertices will be treated in Section 5 as Propositions 5.1 and 5.2. The summation and average of all $F_{j}(n, x)$ for a given stage $n$ will be studied in Section 6, and the average in the infinite $n$ limit will be obtained as Theorem 6.1 .

\section{$3 F_{j}(n, o)$ with $j \in\{1,2\}$}

Consider the Sierpinski gasket $S G(n)$ at stage $n$. We will derive $F_{j}(n, x)$ for the vertex $x=o$ in this section. Since the leftmost vertex $o$ has degree two, $f_{j}(n, o)=0$ for $j=3,4$ and any $n \geq 0$. Similarly, we only need $i \in\{0,1,2\}$ for $g_{i}(n, x)$ and $h_{i}(n, x)$ with $x \in\left\{o, a_{n}, b_{n}\right\}$. Due to the symmetry of $S G(n)$, we have $f_{j}(n, o)=f_{j}\left(n, a_{n}\right)=f_{j}\left(n, b_{n}\right), g_{j}(n, o)=g_{j}\left(n, a_{n}\right)$ with $j=1,2$, and $h_{i}(n, o)=h_{i}\left(n, a_{n}\right)=$ $h_{i}\left(n, b_{n}\right)$ with $i=0,1,2$. According to the definition, $g_{0}(n, o)=g_{0}\left(n, a_{n}\right)=0$, but $g_{0}\left(n, b_{n}\right) \neq 0$ for any $n \geq 0$. In fact, $g_{0}\left(n, b_{n}\right)$ is the only $g_{0}(n, x)$ with non-zero value, and $h_{0}(n, x)$ is non-zero only when $x \in\left\{o, a_{n}, b_{n}\right\}$. The initial values for $x=o$ at stage $n=0$ are $f(0)=3$ with decompositions $f_{1}(0, o)=2$ and $f_{2}(0, o)=1, g(0)=1$ with decompositions $g_{1}(0, o)=1$ and $g_{2}(0, o)=0, h(0)=1$ with decompositions $h_{0}(0, o)=1$ and $h_{j}(0, o)=0$ for $j=1,2$. We also have $g_{0}\left(0, b_{0}\right)=1$ and $g_{j}\left(0, b_{0}\right)=0$ for $j=1,2$.

The following recursion relations were derived in [Chang et al.(2007)] for $n \geq 0$,

$$
\left\{\begin{array}{l}
f(n+1)=6 f(n)^{2} g(n) \\
g(n+1)=f(n)^{2} h(n)+7 f(n) g(n)^{2} \\
h(n+1)=12 f(n) g(n) h(n)+14 g(n)^{3}
\end{array}\right.
$$

as illustrated in Figs. 3 5. $f(n), g(n), h(n)$ were solved exactly in [Chang et al.(2007)] such that they satisfy the relation $3 g(n)^{2}=f(n) h(n)$. It follows that the second and third lines of $(1)$ can be simplified to

$$
\left\{\begin{array}{l}
g(n+1)=10 f(n) g(n)^{2}=\frac{10}{3} f(n)^{2} h(n) \\
h(n+1)=50 g(n)^{3}=\frac{50}{3} f(n) g(n) h(n)
\end{array}\right.
$$


156

Shu-Chiuan Chang and Lung-Chi Chen

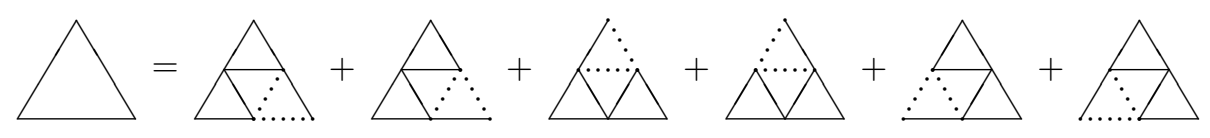

Fig. 3: Illustration for the expression of $f(n+1)$.

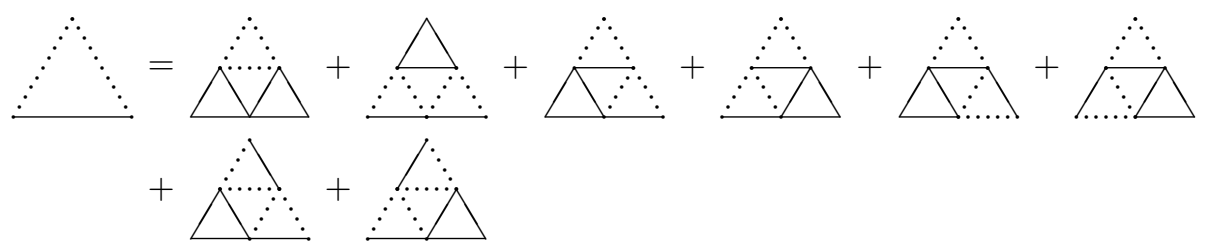

Fig. 4: Illustration for the expression of $g(n+1)$.

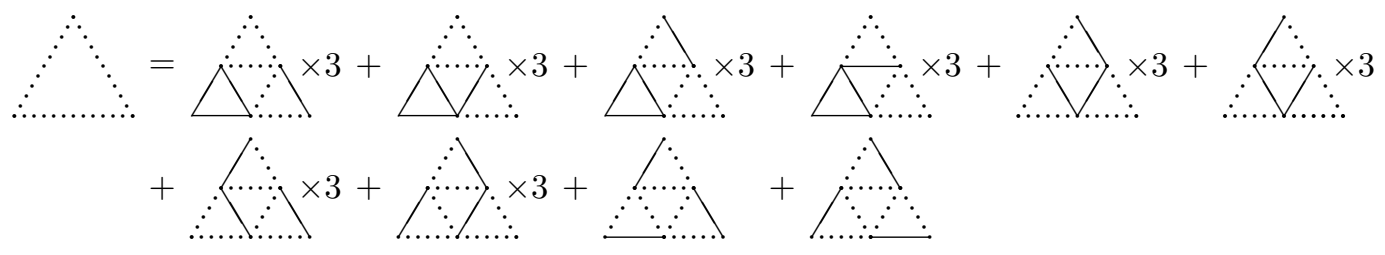

Fig. 5: Illustration for the expression of $h(n+1)$. The multiplication for the eight configurations on the right-hand-side corresponds to three possible orientations. 
Using Figs. 35 for vertex $o$, we obtain the following recursion relations for $j=1,2$ :

$$
\left\{\begin{array}{l}
f_{j}(n+1, o)=4 f_{j}(n, o) f(n) g(n)+2 g_{j}(n, o) f(n)^{2} \\
g_{j}(n+1, o)=f_{j}(n, o) f(n) h(n)+3 f_{j}(n, o) g(n)^{2}+4 g_{j}(n, o) f(n) g(n)
\end{array}\right.
$$

and

$$
\left\{\begin{aligned}
g_{j}\left(n+1, b_{n+1}\right)= & h_{j}(n, o) f(n)^{2}+f_{j}(n, o) g(n)^{2}+2\left[g_{j}(n, o)+2 g_{j}\left(n, b_{n}\right)\right] f(n) g(n), \\
h_{j}(n+1, o)= & 4 h_{j}(n, o) f(n) g(n)+6 g_{j}\left(n, b_{n}\right) g(n)^{2}+4 f_{j}(n, o) g(n) h(n) \\
& +8 g_{j}(n, o) g(n)^{2}+2\left[g_{j}(n, o)+g_{j}\left(n, b_{n}\right)\right] f(n) h(n) .
\end{aligned}\right.
$$

Setting $f_{0}(n, o)=0$ and $g_{0}(n, o)=0,4$ reduces to

$$
\left\{\begin{array}{l}
g_{0}\left(n+1, b_{n+1}\right)=h_{0}(n, o) f(n)^{2}+4 g_{0}\left(n, b_{n}\right) f(n) g(n), \\
h_{0}(n+1, o)=4 h_{0}(n, o) f(n) g(n)+6 g_{0}\left(n, b_{n}\right) g(n)^{2}+2 g_{0}\left(n, b_{n}\right) f(n) h(n) .
\end{array}\right.
$$

The initial values for the probabilities are

$$
\left\{\begin{array}{l}
F_{1}(0, o)=2 / 3, F_{2}(0, o)=1 / 3, G_{1}(0, o)=1, G_{2}(0, o)=0 \\
H_{0}(0, o)=1, G_{0}\left(0, b_{0}\right)=1, G_{j}\left(0, b_{0}\right)=H_{j}(0, o)=0 \text { with } j=1,2 .
\end{array}\right.
$$

Divide the quantities in (3)-(5) by $f(n+1), g(n+1)$ or $h(n+1)$ given in (1) or (2), we get

$$
\begin{gathered}
\left\{\begin{array}{l}
F_{j}(n+1, o)=\frac{2}{3} F_{j}(n, o)+\frac{1}{3} G_{j}(n, o), \\
G_{j}(n+1, o)=\frac{3}{5} F_{j}(n, o)+\frac{2}{5} G_{j}(n, o),
\end{array}\right. \\
\begin{cases}G_{j}\left(n+1, b_{n+1}\right) & =\frac{2}{5} G_{j}\left(n, b_{n}\right)+\frac{3}{10} H_{j}(n, o)+\frac{1}{10} F_{j}(n, o)+\frac{1}{5} G_{j}(n, o), \\
H_{j}(n+1, o) & =\frac{6}{25} G_{j}\left(n, b_{n}\right)+\frac{6}{25} H_{j}(n, o)+\frac{6}{25} F_{j}(n, o)+\frac{7}{25} G_{j}(n, o),\end{cases}
\end{gathered}
$$

for $j=1,2$, and

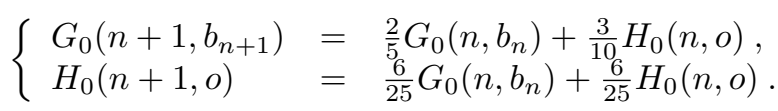

The probabilities $F_{j}(n, o)$ and $G_{j}(n, o)$ with $j=1,2$ can be solved exactly by linear algebra with recursion relations (7) and the initial values given in (6).

Theorem 3.1 For the Sierpinski gasket $S G(n)$ with non-negative integer $n$,

$$
\begin{aligned}
& F_{1}(n, o)=\frac{11}{14}-\frac{5}{42}\left(\frac{1}{15}\right)^{n}, \quad G_{1}(n, o)=\frac{11}{14}+\frac{3}{14}\left(\frac{1}{15}\right)^{n}, \\
& F_{2}(n, o)=\frac{3}{14}+\frac{5}{42}\left(\frac{1}{15}\right)^{n}, \quad G_{2}(n, o)=\frac{3}{14}-\frac{3}{14}\left(\frac{1}{15}\right)^{n} .
\end{aligned}
$$

From Theorem 3.1 and the exact expressions of $f(n), g(n), h(n)$ in [Chang et al.(2007)], we have the following corollary. 
Corollary 3.1 For the Sierpinski gasket $S G(n)$ with non-negative integer n,

$$
\begin{aligned}
& f_{1}(n, o)=\left[\frac{11}{14}-\frac{5}{42}\left(\frac{1}{15}\right)^{n}\right]\left[2^{\alpha(n)} 3^{\beta(n)} 5^{\gamma(n)}\right], \\
& f_{2}(n, o)=\left[\frac{3}{14}+\frac{5}{42}\left(\frac{1}{15}\right)^{n}\right]\left[2^{\alpha(n)} 3^{\beta(n)} 5^{\gamma(n)}\right],
\end{aligned}
$$

where $\alpha(n)=\frac{1}{2}\left(3^{n}-1\right), \beta(n)=\frac{1}{4}\left(3^{n+1}+2 n+1\right)$ and $\gamma(n)=\frac{1}{4}\left(3^{n}-2 n-1\right)$. The limiting probabilities for the vertex o are

$$
\lim _{n \rightarrow \infty} F_{1}(n, o)=\frac{11}{14}, \quad \lim _{n \rightarrow \infty} F_{2}(n, o)=\frac{3}{14} .
$$

In order to derive the probability $F_{j}(n, x)$ for an arbitrary vertex $x \neq o$, we need the following lemma:

Lemma 3.1 For the Sierpinski gasket $S G(n)$ with non-negative integer n,

$$
\begin{aligned}
G_{0}\left(n, b_{n}\right) & =\frac{33}{28}\left(\frac{3}{5}\right)^{n}-\frac{5}{28}\left(\frac{1}{25}\right)^{n}, \quad H_{0}(n, o)=\frac{11}{14}\left(\frac{3}{5}\right)^{n}+\frac{3}{14}\left(\frac{1}{25}\right)^{n}, \\
G_{1}\left(n, b_{n}\right) & =\frac{11}{14}-\frac{2}{7}\left(\frac{1}{15}\right)^{n}-\frac{6}{7}\left(\frac{3}{5}\right)^{n}+\frac{5}{14}\left(\frac{1}{25}\right)^{n}, \\
G_{2}\left(n, b_{n}\right) & =\frac{3}{14}+\frac{2}{7}\left(\frac{1}{15}\right)^{n}-\frac{9}{28}\left(\frac{3}{5}\right)^{n}-\frac{5}{28}\left(\frac{1}{25}\right)^{n}, \\
H_{1}(n, o) & =\frac{11}{14}+\frac{3}{14}\left(\frac{1}{15}\right)^{n}-\frac{4}{7}\left(\frac{3}{5}\right)^{n}-\frac{3}{7}\left(\frac{1}{25}\right)^{n}, \\
H_{2}(n, o) & =\frac{3}{14}-\frac{3}{14}\left(\frac{1}{15}\right)^{n}-\frac{3}{14}\left(\frac{3}{5}\right)^{n}+\frac{3}{14}\left(\frac{1}{25}\right)^{n} .
\end{aligned}
$$

The proof of this lemma can be found in the online version of this paper [Chang and Chen].

$$
4 F_{j}\left(n+m+1, x_{n}\right) \text { with } x \in\{a, b, c\} \text { and } n \geq 0, m \geq 0
$$

Consider the Sierpinski gasket $S G(n+m+1)$ with $n \geq 0, m \geq 0$. We will derive $F_{j}\left(n+1, x_{n}\right)$ with $j \in\{1,2,3,4\}$ for the vertex $x_{n} \in\left\{a_{n}, b_{n}, c_{n}\right\}$ first, then $F_{j}\left(n+m+1, x_{n}\right)$ with arbitrary $m>0$ in this section. The corresponding $G_{j}\left(n+1, x_{n}\right)$ and $H_{j}\left(n+1, x_{n}\right)$ with $j \in\{1,2,3,4\}$ will be used in the next section. Notice that $G_{0}\left(n+1, x_{n}\right)=H_{0}\left(n+1, x_{n}\right)=0$ for $x_{n} \in\left\{a_{n}, b_{n}, c_{n}\right\}$ as these vertices are not outmost vertices of $S G(n+1)$.

For the Sierpinski gasket $S G(n+1)$, we know $f_{j}\left(n+1, a_{n}\right)=f_{j}\left(n+1, b_{n}\right)=f_{j}\left(n+1, c_{n}\right)$ and $h_{j}\left(n+1, a_{n}\right)=h_{j}\left(n+1, b_{n}\right)=h_{j}\left(n+1, c_{n}\right)$ with $j \in\{1,2,3,4\}$ because of rotation symmetry. From the definition of $g_{j}(n, x)$, we have $g_{j}\left(n+1, b_{n}\right)=g_{j}\left(n+1, c_{n}\right)$ but they are distinct from $g_{j}\left(n+1, a_{n}\right)$. The recursion relations for $f_{j}\left(n+1, a_{n}\right), g_{j}\left(n+1, a_{n}\right), g_{j}\left(n+1, b_{n}\right), h_{j}\left(n+1, a_{n}\right)$ with $j \in\{1,2,3,4\}$, and the corresponding $F_{j}\left(n+1, a_{n}\right), G_{j}\left(n+1, a_{n}\right), G_{j}\left(n+1, b_{n}\right), H_{j}\left(n+1, a_{n}\right)$ are lengthy and given in the appendix.

Next consider the Sierpinski gasket $S G(n+m+1)$ with $n \geq 0$ and $m>0$. The left-hand-sides of Figs. 3. 5now represent $S G(n+m+1)$ with positive integer $m$, such that $x_{n} \in\left\{a_{n}, b_{n}, c_{n}\right\}$ locates within the lower-left triangle representing $S G(n+m)$ in the right-hand-sides of the figures. As the vertices are 
denoted such that $x_{\vec{\gamma}}$ and $\tilde{x}_{\vec{\gamma}}$ are reflection of each other with respect to the extended line connecting $o$ and $c_{0}$, we have $\tilde{a}_{n}=b_{n}, \tilde{b}_{n}=a_{n}$ and $\tilde{c}_{n}=c_{n}$. We obtain the following recursion relations for $j \in\{1,2,3,4\}$ :

$$
\begin{aligned}
F_{j}\left(n+m+1, x_{n}\right)= & \frac{4 f_{j}\left(n+m, x_{n}\right) f(n+m) g(n+m)}{6 f(n+m)^{2} g(n+m)} \\
& +\frac{\left[g_{j}\left(n+m, x_{n}\right)+g_{j}\left(n+m, \tilde{x}_{n}\right)\right] f(n+m)^{2}}{6 f(n+m)^{2} g(n+m)} \\
= & \frac{2 F_{j}\left(n+m, x_{n}\right)}{3}+\frac{G_{j}\left(n+m, x_{n}\right)+G_{j}\left(n+m, \tilde{x}_{n}\right)}{6},
\end{aligned}
$$

and

$$
G_{j}\left(n+m+1, x_{n}\right)=\frac{3 F_{j}\left(n+m, x_{n}\right)}{5}+\frac{3 G_{j}\left(n+m, x_{n}\right)}{10}+\frac{G_{j}\left(n+m, \tilde{x}_{n}\right)}{10} .
$$

By symmetry, we know $F_{j}\left(n+m, \tilde{x}_{n}\right)=F_{j}\left(n+m, x_{n}\right)$ with $x_{n} \in\left\{a_{n}, b_{n}, c_{n}\right\}$ and $G_{j}\left(n+m, \tilde{c}_{n}\right)=$ $G_{j}\left(n+m, c_{n}\right)$ for positive integer $m$. Let us define the $3 \times 3$ matrix

$$
B_{j}^{\prime}(n+m+1, n)=\left[\begin{array}{lll}
F_{j}\left(n+m+1, a_{n}\right) & G_{j}\left(n+m+1, a_{n}\right) & G_{j}\left(n+m+1, b_{n}\right) \\
F_{j}\left(n+m+1, b_{n}\right) & G_{j}\left(n+m+1, b_{n}\right) & G_{j}\left(n+m+1, a_{n}\right) \\
F_{j}\left(n+m+1, c_{n}\right) & G_{j}\left(n+m+1, c_{n}\right) & G_{j}\left(n+m+1, c_{n}\right)
\end{array}\right]
$$

for non-negative integer $n, m$ and $j \in\{1,2,3,4\}$. For $m=0, B_{j}^{\prime}(n+1, n)$ has been obtained with elements given by (36)-38) in the appendix. By (10) and (11), we have

$$
B_{j}^{\prime}(n+m+1, n)=B_{j}^{\prime}(n+m, n) L^{\prime},
$$

for any $m \geq 1$, where

$$
L^{\prime}=\left[\begin{array}{ccc}
\frac{2}{3} & \frac{3}{5} & \frac{3}{5} \\
\frac{1}{6} & \frac{3}{10} & \frac{1}{10} \\
\frac{1}{6} & \frac{1}{10} & \frac{3}{10}
\end{array}\right]
$$

We arrive at

$$
B_{j}^{\prime}(n+m+1, n)=B_{j}^{\prime}(n+1, n) L^{\prime m} \quad \text { for all } m \geq 0, n \geq 0 .
$$

Solving $B_{j}^{\prime}(n+m+1, n)$ as in the proof of Theorem 3.1, its first column gives

$$
\begin{aligned}
{\left[\begin{array}{c}
F_{j}\left(n+m+1, a_{n}\right) \\
F_{j}\left(n+m+1, b_{n}\right) \\
F_{j}\left(n+m+1, c_{n}\right)
\end{array}\right]=} & {\left[\frac{9}{14}+\frac{5}{14}\left(\frac{1}{15}\right)^{m}\right]\left[\begin{array}{c}
F_{j}\left(n+1, a_{n}\right) \\
F_{j}\left(n+1, b_{n}\right) \\
F_{j}\left(n+1, c_{n}\right)
\end{array}\right] } \\
& +\frac{5}{28}\left[1-\left(\frac{1}{15}\right)^{m}\right]\left[\begin{array}{c}
G_{j}\left(n+1, a_{n}\right)+G_{j}\left(n+1, b_{n}\right) \\
G_{j}\left(n+1, b_{n}\right)+G_{j}\left(n+1, a_{n}\right) \\
2 G_{j}\left(n+1, c_{n}\right)
\end{array}\right],
\end{aligned}
$$

and we have the following theorem using (36)-38]. 
Theorem 4.1 For the Sierpinski gasket $S G(n+m+1)$ with non-negative integer $n$ and $m$,

$$
\begin{aligned}
& \left\{\begin{array}{l}
F_{1}\left(n+m+1, a_{n}\right)=\frac{1815}{5488}\left(\frac{3}{5}\right)^{n}-\frac{99}{1372}\left(\frac{1}{25}\right)^{n}+\frac{55}{16464}\left(\frac{1}{375}\right)^{n} \\
\quad+\left(\frac{1}{15}\right)^{m}\left\{-\frac{121}{5488}\left(\frac{3}{5}\right)^{n}-\frac{22}{1029}\left(\frac{1}{25}\right)^{n}+\frac{185}{49392}\left(\frac{1}{375}\right)^{n}\right\} \\
F_{2}\left(n+m+1, a_{n}\right)=\frac{121}{196}-\frac{825}{5488}\left(\frac{3}{5}\right)^{n}+\frac{171}{1372}\left(\frac{1}{25}\right)^{n}-\frac{55}{5488}\left(\frac{1}{375}\right)^{n}-\frac{121}{1176}\left(\frac{1}{15}\right)^{n}+\frac{1}{294}\left(\frac{1}{225}\right)^{n} \\
\quad+\left(\frac{1}{15}\right)^{m}\left\{\frac{55}{5488}\left(\frac{3}{5}\right)^{n}+\frac{38}{1029}\left(\frac{1}{25}\right)^{n}-\frac{185}{16464}\left(\frac{1}{375}\right)^{n}-\frac{143}{3528}\left(\frac{1}{15}\right)^{n}+\frac{11}{2646}\left(\frac{1}{225}\right)^{n}\right\} \\
\quad F_{3}\left(n+m+1, a_{n}\right)=\frac{33}{98}-\frac{855}{5488}\left(\frac{3}{5}\right)^{n}-\frac{45}{1372}\left(\frac{1}{25}\right)^{n}+\frac{55}{5488}\left(\frac{1}{375}\right)^{n}+\frac{11}{147}\left(\frac{1}{15}\right)^{n}-\frac{1}{147}\left(\frac{1}{225}\right)^{n} \\
\quad+\left(\frac{1}{15}\right)^{m}\left\{\frac{57}{5488}\left(\frac{3}{5}\right)^{n}-\frac{10}{1029}\left(\frac{1}{25}\right)^{n}+\frac{185}{16464}\left(\frac{1}{375}\right)^{n}+\frac{13}{441}\left(\frac{1}{15}\right)^{n}-\frac{11}{1323}\left(\frac{1}{225}\right)^{n}\right\} \\
\quad F_{4}\left(n+m+1, a_{n}\right)=\frac{9}{196}-\frac{135}{5488}\left(\frac{3}{5}\right)^{n}-\frac{27}{1372}\left(\frac{1}{25}\right)^{n}-\frac{55}{16464}\left(\frac{1}{375}\right)^{n}+\frac{11}{392}\left(\frac{1}{15}\right)^{n}+\frac{1}{294}\left(\frac{1}{225}\right)^{n} \\
\quad+\left(\frac{1}{15}\right)^{m}\left\{\frac{9}{5488}\left(\frac{3}{5}\right)^{n}-\frac{2}{343}\left(\frac{1}{25}\right)^{n}-\frac{185}{49392}\left(\frac{1}{375}\right)^{n}+\frac{13}{1176}\left(\frac{1}{15}\right)^{n}+\frac{11}{2646}\left(\frac{1}{225}\right)^{n}\right\}
\end{array}\right. \\
& \left\{\begin{array}{l}
F_{1}\left(n+m+1, c_{n}\right)=\frac{1089}{2744}\left(\frac{3}{5}\right)^{n}-\frac{22}{343}\left(\frac{1}{25}\right)^{n}+\frac{5}{8232}\left(\frac{1}{375}\right)^{n} \\
\quad+\left(\frac{1}{15}\right)^{m}\left\{-\frac{121}{1372}\left(\frac{3}{5}\right)^{n}-\frac{121}{4116}\left(\frac{1}{25}\right)^{n}+\frac{20}{3087}\left(\frac{1}{375}\right)^{n}\right\} \\
F_{2}\left(n+m+1, c_{n}\right)=\frac{121}{196}-\frac{495}{2744}\left(\frac{3}{5}\right)^{n}+\frac{38}{343}\left(\frac{1}{25}\right)^{n}-\frac{5}{2744}\left(\frac{1}{375}\right)^{n}-\frac{55}{588}\left(\frac{1}{15}\right)^{n} \\
\quad+\left(\frac{1}{15}\right)^{m}\left\{\frac{55}{1372}\left(\frac{3}{5}\right)^{n}+\frac{209}{4116}\left(\frac{1}{25}\right)^{n}-\frac{20}{1029}\left(\frac{1}{375}\right)^{n}-\frac{22}{441}\left(\frac{1}{15}\right)^{n}+\frac{10}{1323}\left(\frac{1}{225}\right)^{n}\right\} \\
F_{3}\left(n+m+1, c_{n}\right)=\frac{33}{98}-\frac{513}{2744}\left(\frac{3}{5}\right)^{n}-\frac{10}{343}\left(\frac{1}{25}\right)^{n}+\frac{5}{2744}\left(\frac{1}{375}\right)^{n}+\frac{10}{147}\left(\frac{1}{15}\right)^{n} \\
\quad+\left(\frac{1}{15}\right)^{m}\left\{\frac{57}{1372}\left(\frac{3}{5}\right)^{n}-\frac{55}{4116}\left(\frac{1}{25}\right)^{n}+\frac{20}{1029}\left(\frac{1}{375}\right)^{n}+\frac{16}{441}\left(\frac{1}{15}\right)^{n}-\frac{20}{1323}\left(\frac{1}{225}\right)^{n}\right\} \\
F_{4}\left(n+m+1, c_{n}\right)=\frac{9}{196}-\frac{81}{2744}\left(\frac{3}{5}\right)^{n}-\frac{6}{343}\left(\frac{1}{25}\right)^{n}-\frac{5}{8232}\left(\frac{1}{375}\right)^{n}+\frac{5}{196}\left(\frac{1}{15}\right)^{n} \\
\quad+\left(\frac{1}{15}\right)^{m}\left\{\frac{9}{1372}\left(\frac{3}{5}\right)^{n}-\frac{11}{1372}\left(\frac{1}{25}\right)^{n}-\frac{20}{3087}\left(\frac{1}{375}\right)^{n}+\frac{2}{147}\left(\frac{1}{15}\right)^{n}+\frac{10}{1323}\left(\frac{1}{225}\right)^{n}\right\}
\end{array}\right.
\end{aligned}
$$

Corollary 4.1 For the Sierpinski gasket $S G(n+m+1)$ with non-negative integer $n$ and $m$, the limiting probabilities are

$$
\begin{array}{ll}
\lim _{n \rightarrow \infty} F_{1}\left(n+m+1, x_{n}\right)=0, & \lim _{n \rightarrow \infty} F_{2}\left(n+m+1, x_{n}\right)=\frac{121}{196}, \\
\lim _{n \rightarrow \infty} F_{3}\left(n+m+1, x_{n}\right)=\frac{33}{98}, & \lim _{n \rightarrow \infty} F_{4}\left(n+m+1, x_{n}\right)=\frac{9}{196},
\end{array}
$$

where the vertex $x_{n}$ can be either $a_{n}, b_{n}$ or $c_{n}$.

It is intriguing to notice that in Theorem $4.1 . F_{j}\left(n+m+1, a_{n}\right)$ are distinct from $F_{j}\left(n+m+1, c_{n}\right)$ with $j \in\{1,2,3,4\}$, while they have the same value in the infinite $n$ limit.

\section{$5 F_{j}(n+m, x)$ for general $x_{\vec{\gamma}} \in V(S G(n))$ with $n \geq 0, m \geq 0$}

Consider the Sierpinski gasket $S G(n+m)$ with $n \geq 0, m \geq 0$. We will derive in this section $F_{j}(n+m, x)$ with $j \in\{1,2,3,4\}$ for the general vertex $x_{\vec{\gamma}} \in V(S G(n))$ that has not been considered in previous sections. For the vertices inside the triangle with outmost vertices $a_{n-1}, a_{n}$ and $c_{n-1}$, let us append subscripts in the notation such that $\vec{\gamma}_{n, s}=\left(\gamma_{1}=n-1, \gamma_{2}, \cdots, \gamma_{s}\right)$ with $1 \leq s \leq n$ and $\gamma_{k} \in\{0,1,2\}$ for $k \in\{2,3, \ldots, s\}$. The results obtained in section 4 correspond to the vertices with $s=1$ and $n \geq 1$, and we will tackle the vertices with $s>1$ here. Similar to the definition of the vertex $\tilde{x}_{\vec{\gamma}_{n, s}}$, let us define the vertex $\hat{x}_{\vec{\gamma}_{n, s}}$ as the reflection of $x_{\vec{\gamma}_{n, s}}$ with respect to the line connecting $a_{n}$ and $b_{n-1}$. By definition, we have

$$
\tilde{\tilde{x}}_{\vec{\gamma}_{n, s}}=x_{\vec{\gamma}_{n, s}}, \hat{\hat{x}}_{\vec{\gamma}_{n, s}}=x_{\vec{\gamma}_{n, s}}
$$


where $x$ can be either $a, b$ or $c$, and

$$
F_{j}\left(n, x_{\vec{\gamma}_{n, s}}\right)=F_{j}\left(n, \tilde{x}_{\vec{\gamma}_{n, s}}\right)=F_{j}\left(n, \hat{x}_{\vec{\gamma}_{n, s}}\right), \quad H_{j}\left(n, x_{\vec{\gamma}_{n, s}}\right)=H_{j}\left(n, \tilde{x}_{\vec{\gamma}_{n, s}}\right)=H_{j}\left(n, \hat{x}_{\vec{\gamma}_{n, s}}\right),
$$

due to the symmetry of $S G(n)$. For $m \geq 0$, define the $3 \times 5$ matrix

$$
\begin{aligned}
& B_{j}\left(n+m, \vec{\gamma}_{n, s}\right) \\
& =\left[\begin{array}{ccccc}
F_{j}\left(n+m, a_{\vec{\gamma}_{n, s}}\right) & G_{j}\left(n+m, a_{\vec{\gamma}_{n, s}}\right) & G_{j}\left(n+m, \tilde{a}_{\vec{\gamma}_{n, s}}\right) & G_{j}\left(n+m, \hat{a}_{\vec{\gamma}_{n, s}}\right) & H_{j}\left(n+m, a_{\vec{\gamma}_{n, s}}\right) \\
F_{j}\left(n+m, b_{\vec{\gamma}_{n, s}}\right) & G_{j}\left(n+m, b_{\vec{\gamma}_{n, s}}\right) & G_{j}\left(n+m, \hat{b}_{\vec{\gamma}_{n, s}}\right) & G_{j}\left(n+m, \hat{b}_{\vec{\gamma}_{n, s}}\right) & H_{j}\left(n+m, b_{\vec{\gamma}_{n, s}}\right) \\
F_{j}\left(n+m, c_{\vec{\gamma}_{n, s}}\right) & G_{j}\left(n+m, c_{\vec{\gamma}_{n, s}}\right) & G_{j}\left(n+m, \tilde{c}_{\vec{\gamma}_{n, s}}\right) & G_{j}\left(n+m, \hat{c}_{\vec{\gamma}_{n, s}}\right) & H_{j}\left(n+m, c_{\vec{\gamma}_{n, s}}\right)
\end{array}\right] .
\end{aligned}
$$

This is a generalization of $B_{j}^{\prime}(n+m, n)$ in $\sqrt{12}$, which corresponds to the case with $s=1$. By an argument similar to that of (14), we have

$$
B_{j}\left(n+m, \gamma_{n, s}\right)=B_{j}\left(n, \gamma_{n, s}\right) L^{m}
$$

for $m \geq 0$, where the $5 \times 5$ matrix

$$
L=\left[\begin{array}{ccccc}
\frac{2}{3} & \frac{3}{5} & \frac{3}{5} & \frac{3}{5} & \frac{6}{25} \\
\frac{1}{6} & \frac{3}{10} & \frac{1}{10} & 0 & \frac{7}{50} \\
\frac{1}{6} & \frac{1}{10} & \frac{3}{10} & \frac{1}{10} & \frac{7}{50} \\
0 & 0 & 0 & \frac{3}{10} & \frac{6}{25} \\
0 & 0 & 0 & 0 & \frac{6}{25}
\end{array}\right]
$$

is the generalization of $L^{\prime}$ in $\left[13\right.$. It follows that the determination of $B_{j}\left(n, \vec{\gamma}_{n, s}\right)$ for $s>1$ will be sufficient.

Let us first consider the vertices with $s=2$ and $\gamma_{2}=1$, namely, $\vec{\gamma}_{n, 2}=(n-1,1)$ with $n=2,3, \ldots$ We obtain the following equations (cf. Figs. 3, 5):

$$
\left\{\begin{aligned}
F_{j}\left(n, x_{(n-1,1)}\right)= & \frac{2 F_{j}\left(n-1, x_{n-2}\right)}{3}+\frac{G_{j}\left(n-1, x_{n-2}\right)}{6}+\frac{G_{j}\left(n-1, \hat{x}_{n-2}\right)}{6}, \\
G_{j}\left(n, x_{(n-1,1)}\right)= & \frac{3 F_{j}\left(n-1, x_{n-2}\right)}{5}+\frac{3 G_{j}\left(n-1, x_{n-2}\right)}{10}+\frac{G_{j}\left(n-1, \hat{x}_{n-2}\right)}{10} \\
G_{j}\left(n, \tilde{x}_{(n-1,1)}\right)= & \frac{3 H_{j}\left(n-1, x_{n-2}\right)}{10}+\frac{F_{j}\left(n-1, x_{n-2}\right)}{10}+\frac{2 G_{j}\left(n-1, \tilde{x}_{n-2}\right)}{5} \\
& +\frac{G_{j}\left(n-1, x_{n-2}\right)}{10}+\frac{G_{j}\left(n-1, \hat{x}_{n-2}\right)}{10} \\
G_{j}\left(n, \hat{x}_{(n-1,1)}\right)= & \frac{3 F_{j}\left(n-1, x_{n-2}\right)}{5}+\frac{3 G_{j}\left(n-1, \hat{x}_{n-2}\right)}{10}+\frac{G_{j}\left(n-1, x_{n-2}\right)}{10} \\
H_{j}\left(n+1, x_{(n-1,1)}\right)= & \frac{6 F_{j}\left(n-1, x_{n-2}\right)}{25}+\frac{7 G_{j}\left(n-1, x_{n-2}\right)}{50}+\frac{6 G_{j}\left(n-1, \tilde{x}_{n-2}\right)}{25} \\
& +\frac{7 G_{j}\left(n-1, \hat{x}_{n-2}\right)}{50}+\frac{6 H_{j}\left(n-1, x_{n-2}\right)}{25}
\end{aligned}\right.
$$

where $x$ can be either $a, b$ or $c$. Define the $5 \times 5$ matrix

$$
R=\left[\begin{array}{ccccc}
\frac{2}{3} & \frac{3}{5} & \frac{1}{10} & \frac{3}{5} & \frac{6}{25} \\
\frac{1}{6} & \frac{3}{10} & \frac{1}{10} & \frac{1}{10} & \frac{7}{50} \\
0 & 0 & \frac{2}{5} & 0 & \frac{6}{25} \\
\frac{1}{6} & \frac{1}{10} & \frac{1}{10} & \frac{3}{10} & \frac{7}{50} \\
0 & 0 & \frac{3}{10} & 0 & \frac{6}{25}
\end{array}\right],
$$


then 16 is equivalent to

$$
B_{j}(n,(n-1,1))=B_{j}(n-1, n-2) R .
$$

For general $m \geq 0$, we have the following formula combining (15) and (18):

$$
B_{j}(n+m,(n-1,1))=B_{j}(n,(n-1,1)) L^{m}=B_{j}(n-1, n-2) R L^{m} .
$$

As $F_{j}\left(n+m, x_{n-1,1}\right)=F_{j}\left(n+m, \tilde{x}_{n-1,1}\right)$ for $x=a, b, c$, the first column of the matrix in 19 gives all $F_{j}\left(n+m, x_{\vec{\gamma}_{n, 2}}\right)$ in terms of the quantities for $x_{n-2}$.

Proposition 5.1 For the Sierpinski gasket $S G(n+m)$ with $n \geq 2, m \geq 0$,

$$
\left[\begin{array}{c}
F_{j}\left(n+m, a_{n-1,1}\right) \\
F_{j}\left(n+m, b_{n-1,1}\right) \\
F_{j}\left(n+m, c_{n-1,1}\right)
\end{array}\right]=\left[\begin{array}{c}
F_{j}\left(n+m, \tilde{a}_{n-1,1}\right) \\
F_{j}\left(n+m, \tilde{b}_{n-1,1}\right) \\
F_{j}\left(n+m, \tilde{c}_{n-1,1}\right)
\end{array}\right]=B_{j}(n-1, n-2) R L^{m} e_{1},
$$

where

$$
e_{1}=(1,0,0,0,0)^{T}
$$

and $j \in\{1,2,3,4\}$.

Move on to the general vertex $x_{\vec{\gamma}_{n+1, s}}$ inside the triangle with outmost vertices $a_{n}, a_{n+1}$ and $c_{n}$ for the Sierpinski gasket $S G(n+1)$, where $\vec{\gamma}_{n+1, s}=\left(n, 1, \gamma_{3}, \ldots, \gamma_{s}\right)$ with $\gamma_{k} \in\{0,1,2\}, k=3,4, . ., s$ and $3 \leq s \leq n+1$. As $\gamma_{3}$ can take three possible values, let us discuss them separately.

First consider the case with $\gamma_{3}=1$. The vertex $x_{\vec{\gamma}_{n+1, s}}=x_{\left(n, 1,1, \gamma_{4}, \ldots \gamma_{s}\right)}$ is located inside the triangle with outmost vertices $a_{n, 1}, a_{n+1}$ and $c_{n, 1}$. Associate with this $x_{\vec{\gamma}_{n+1, s}}$ a vertex $x_{\vec{\gamma}_{n, s-1}^{1}}$, where $\vec{\gamma}_{n, s-1}^{1}=$ $\left(n-1,1, \gamma_{4}, \ldots, \gamma_{s}\right)$ has $s-1$ components. That is, $\vec{\gamma}_{n, s-1}^{1}$ is obtained from $\vec{\gamma}_{n+1, s}$ by taking out $\gamma_{3}=1$ and replacing $\gamma_{1}=n$ by $n-1$. It can be seen that this vertex $x_{\vec{\gamma}_{n, s-1}^{1}}$ is located inside the triangle with outmost vertices $a_{n-1}, a_{n}$ and $c_{n-1}$. Moreover, $x_{\vec{\gamma}_{n, s-1}^{1}}$ can be reached from $x_{\vec{\gamma}_{n+1, s}}$ by a horizontal translation with the distance from $a_{n}$ to $o$. Particularly, if $s=3$ such that $\vec{\gamma}_{n+1, s}=(n, 1,1)$, then $\vec{\gamma}_{n, s-1}^{1}=(n-1,1)$. By the method obtaining $[18)$, we have

$$
B_{j}\left(n+1, \vec{\gamma}_{n+1, s}\right)=B_{j}\left(n, \vec{\gamma}_{n, s-1}^{1}\right) R
$$

if $\gamma_{3}=1$.

Now consider the case with $\gamma_{3}=2$. The vertex $x_{\vec{\gamma}_{n+1, s}}=x_{\left(n, 1,2, \gamma_{4}, \ldots \gamma_{s}\right)}$ is located inside the triangle with outmost vertices $b_{n, 1}, c_{n, 1}$ and $c_{n}$. Associate with this $x_{\vec{\gamma}_{n+1, s}}$ a vertex $\tilde{y}_{\vec{\gamma}_{n, s-1}^{2}}$ where $\vec{\gamma}_{n, s-1}^{2}=$ $\left.\left(n-1,1, \gamma_{4}^{2}, \ldots, \gamma_{s}^{2}\right)\right)$ has $s-1$ components. Here $y=b$ when $x=a$ and vice versa, and $y=c$ when $x=c$. Namely, $y$ is related to $x$ with three possibilities: $(x, y)=(a, b),(b, a)$ and $(c, c)$. Similarly, $\gamma_{k}^{2}$ is related to $\gamma_{k}$ with three possibilities: $\left(\gamma_{k}, \gamma_{k}^{2}\right)=(1,2),(2,1)$ and $(0,0)$, where $k=4, \ldots, s$. Again, $\tilde{y}_{\vec{\gamma}_{n, s-1}^{2}}$ can be reached from $x_{\vec{\gamma}_{n+1, s}}$ by a horizontal translation with the distance from $a_{n}$ to $o$. We have

$$
B_{j}\left(n+1, \vec{\gamma}_{n+1, s}\right)=\tilde{B}_{j}\left(n, \vec{\gamma}_{n, s-1}^{2}\right) R
$$

where

$$
\tilde{B}_{j}\left(n, \vec{\gamma}_{n, s-1}^{2}\right)
$$




$$
=\left[\begin{array}{ccccc}
F_{j}\left(n, \tilde{b}_{\vec{\gamma}_{n, s-1}^{2}}\right) & G_{j}\left(n, \tilde{b}_{\vec{\gamma}_{n, s-1}^{2}}\right) & G_{j}\left(n, \tilde{\tilde{b}}_{\vec{\gamma}_{n, s-1}^{2}}\right) & G_{j}\left(n, \hat{\tilde{b}}_{\vec{\gamma}_{n, s-1}^{2}}\right) & H_{j}\left(n, \tilde{b}_{\vec{\gamma}_{n, s-1}^{2}}\right) \\
F_{j}\left(n, \tilde{a}_{\vec{\gamma}_{n, s-1}^{2}}\right) & G_{j}\left(n, \tilde{a}_{\vec{\gamma}_{n, s-1}^{2}}\right) & G_{j}\left(n, \tilde{\tilde{a}}_{\vec{\gamma}_{n, s-1}^{2}}\right) & G_{j}\left(n, \hat{\tilde{a}}_{\vec{\gamma}_{n, s-1}^{2}}\right) & H_{j}\left(n, \tilde{a}_{\vec{\gamma}_{n, s-1}^{2}}\right) \\
F_{j}\left(n, \tilde{c}_{\vec{\gamma}_{n, s-1}^{2}}\right) & G_{j}\left(n, \tilde{c}_{\vec{\gamma}_{n, s-1}^{2}}\right) & G_{j}\left(n, \tilde{\tilde{c}}_{\vec{\gamma}_{n, s-1}^{2}}\right) & G_{j}\left(n, \hat{\tilde{c}}_{\vec{\gamma}_{n, s-1}^{2}}^{2}\right) & H_{j}\left(n, \tilde{c}_{\vec{\gamma}_{n, s-1}^{2}}\right)
\end{array}\right]
$$

By symmetry, the columns in 22 can be replaced as

$$
\left[\begin{array}{c}
F_{j}\left(n, \tilde{b}_{\vec{\gamma}_{n, s-1}^{2}}\right) \\
F_{j}\left(n, \tilde{a}_{\vec{\gamma}_{n, s-1}^{2}}\right) \\
F_{j}\left(n, \tilde{c}_{\vec{\gamma}_{n, s-1}^{2}}^{2}\right)
\end{array}\right]=\left[\begin{array}{c}
F_{j}\left(n, b_{\vec{\gamma}_{n, s-1}^{2}}\right) \\
F_{j}\left(n, a_{\vec{\gamma}_{n, s-1}^{2}}\right) \\
F_{j}\left(n, c_{\vec{\gamma}_{n, s-1}^{2}}^{2}\right)
\end{array}\right], \quad\left[\begin{array}{c}
H_{j}\left(n, \tilde{b}_{\vec{\gamma}_{n, s-1}^{2}}\right) \\
H_{j}\left(n, \tilde{a}_{\vec{\gamma}_{n, s-1}^{2}}\right) \\
H_{j}\left(n, \tilde{c}_{\vec{\gamma}_{n, s-1}^{2}}\right)
\end{array}\right]=\left[\begin{array}{c}
H_{j}\left(n, b_{\vec{\gamma}_{n, s-1}^{2}}\right) \\
H_{j}\left(n, a_{\vec{\gamma}_{n, s-1}^{2}}\right) \\
H_{j}\left(n, c_{\vec{\gamma}_{n, s-1}^{2}}\right)
\end{array}\right],
$$

and

$$
\left[\begin{array}{c}
G_{j}\left(n, \tilde{\tilde{b}}_{\vec{\gamma}_{n, s-1}^{2}}\right) \\
G_{j}\left(n, \tilde{\tilde{a}}_{\vec{\gamma}_{n, s-1}^{2}}^{2}\right) \\
G_{j}\left(n, \tilde{\tilde{c}}_{\vec{\gamma}_{n, s-1}^{2}}^{2}\right)
\end{array}\right]=\left[\begin{array}{c}
G_{j}\left(n, b_{\vec{\gamma}_{n, s-1}^{2}}\right) \\
G_{j}\left(n, a_{\vec{\gamma}_{n, s-1}^{2}}\right) \\
G_{j}\left(n, c_{\vec{\gamma}_{n, s-1}^{2}}^{2}\right)
\end{array}\right], \quad\left[\begin{array}{c}
G_{j}\left(n, \hat{\tilde{b}}_{\vec{\gamma}_{n, s-1}^{2}}\right) \\
G_{j}\left(n, \hat{\tilde{a}}_{\vec{\gamma}_{n, s-1}^{2}}\right) \\
G_{j}\left(n, \tilde{\tilde{c}}_{\vec{\gamma}_{n, s-1}^{2}}\right)
\end{array}\right]=\left[\begin{array}{c}
G_{j}\left(n, \hat{b}_{\vec{\gamma}_{n, s-1}^{2}}\right) \\
G_{j}\left(n, \hat{a}_{\vec{\gamma}_{n, s-1}^{2}}\right) \\
G_{j}\left(n, \hat{c}_{\vec{\gamma}_{n, s-1}^{2}}^{2}\right)
\end{array}\right],
$$

so that

$$
\tilde{B}_{j}\left(n, \vec{\gamma}_{n, s-1}^{2}\right)=B_{j}\left(n, \vec{\gamma}_{n, s-1}^{2}\right) E_{2}
$$

where

$$
E_{2}=\left[\begin{array}{lllll}
1 & 0 & 0 & 0 & 0 \\
0 & 0 & 1 & 0 & 0 \\
0 & 1 & 0 & 0 & 0 \\
0 & 0 & 0 & 1 & 0 \\
0 & 0 & 0 & 0 & 1
\end{array}\right]
$$

(21) can be rewritten as

$$
B_{j}\left(n+1, \vec{\gamma}_{n+1, s}\right)=B_{j}\left(n, \vec{\gamma}_{n, s-1}^{2}\right) E_{2} R
$$

if $\gamma_{3}=2$.

Finally consider the case with $\gamma_{3}=0$. The vertex $x_{\vec{\gamma}_{n+1, s}}=x_{\left(n, 1,0, \gamma_{4}, \ldots \gamma_{s}\right)}$ is located inside the triangle with outmost vertices $a_{n}, a_{n, 1}$ and $b_{n, 1}$. Associate with this $x_{\vec{\gamma}_{n+1, s}}$ a vertex $\bar{z}_{\vec{\gamma}_{n, s-1}^{0}}$ where $\vec{\gamma}_{n, s-1}^{0}=\left(n-1,1, \gamma_{4}^{0}, \ldots, \gamma_{s}^{0}\right)$ has $s-1$ components. Here $z=c$ when $x=b$ and vice versa, and $z=a$ when $x=a$. Namely, $z$ is related to $x$ with three possibilities: $(x, z)=(a, a),(b, c)$ and $(c, b)$. Similarly, $\gamma_{k}^{0}$ is related to $\gamma_{k}$ with three possibilities: $\left(\gamma_{k}, \gamma_{k}^{0}\right)=(1,0),(0,1)$ and $(2,2)$, where $k=4, \ldots, s$. We use the notation such that the vertex $\bar{z}_{\vec{\gamma}_{n, s-1}^{0}}$ is the reflection of the vertex $x_{\vec{\gamma}_{n, s-1}}$ with respect to the line connecting $a_{n-1}$ and $b_{n}$. It can be seen that $\bar{z}_{\vec{\gamma}_{n, s-1}^{0}}$ can be reached from $x_{\vec{\gamma}_{n+1, s}}$ by a horizontal translation with the distance from $a_{n}$ to $o$. We have

$$
B_{j}\left(n+1, \vec{\gamma}_{n+1, s}\right)=\bar{B}_{j}\left(n, \vec{\gamma}_{n, s-1}^{0}\right) R,
$$

where

$$
\bar{B}_{j}\left(n, \vec{\gamma}_{n, s-1}^{0}\right)
$$




$$
\begin{array}{r}
=\left[\begin{array}{lllll}
F_{j}\left(n, \bar{a}_{\vec{\gamma}_{n, s-1}^{0}}\right) & G_{j}\left(n, \bar{a}_{\vec{\gamma}_{n, s-1}^{0}}\right) & G_{j}\left(n, \tilde{\bar{a}}_{\vec{\gamma}_{n, s-1}^{0}}\right) & G_{j}\left(n, \hat{\bar{a}}_{\vec{\gamma}_{n, s-1}^{0}}\right) & H_{j}\left(n, \bar{a}_{\vec{\gamma}_{n, s-1}^{0}}\right) \\
F_{j}\left(n, \bar{c}_{\vec{\gamma}_{n, s-1}^{0}}\right) & G_{j}\left(n, \bar{c}_{\vec{\gamma}_{n, s-1}^{0}}\right) & G_{j}\left(n, \tilde{\bar{c}}_{\vec{\gamma}_{n, s-1}^{0}}\right) & G_{j}\left(n, \hat{\bar{c}}_{\vec{\gamma}_{n, s-1}^{0}}\right) & H_{j}\left(n, \bar{c}_{\vec{\gamma}_{n, s-1}^{0}}\right) \\
F_{j}\left(n, \bar{b}_{\vec{\gamma}_{n, s-1}^{0}}\right) & G_{j}\left(n, \bar{b}_{\vec{\gamma}_{n, s-1}^{0}}\right) & G_{j}\left(n, \tilde{\bar{b}}_{\vec{\gamma}_{n, s-1}^{0}}\right) & G_{j}\left(n, \hat{\bar{b}}_{\vec{\gamma}_{n, s-1}^{0}}\right) & H_{j}\left(n, \bar{b}_{\vec{\gamma}_{n, s-1}^{0}}\right)
\end{array}\right] \\
=\left[\begin{array}{lllll}
F_{j}\left(n, a_{\vec{\gamma}_{n, s-1}^{0}}\right) & G_{j}\left(n, a_{\vec{\gamma}_{n, s-1}^{0}}\right) & G_{j}\left(n, \hat{a}_{\vec{\gamma}_{n, s-1}^{0}}\right) & G_{j}\left(n, \tilde{a}_{\vec{\gamma}_{n, s-1}^{0}}\right) & H_{j}\left(n, a_{\vec{\gamma}_{n, s-1}^{0}}\right) \\
F_{j}\left(n, c_{\vec{\gamma}_{n, s-1}^{0}}\right) & G_{j}\left(n, c_{\vec{\gamma}_{n, s-1}^{0}}\right) & G_{j}\left(n, \hat{c}_{\vec{\gamma}_{n, s-1}^{0}}\right) & G_{j}\left(n, \tilde{c}_{\vec{\gamma}_{n, s-1}^{0}}\right) & H_{j}\left(n, c_{\vec{\gamma}_{n, s-1}^{0}}\right) \\
F_{j}\left(n, b_{\vec{\gamma}_{n, s-1}^{0}}\right) & G_{j}\left(n, b_{\vec{\gamma}_{n, s-1}^{0}}\right) & G_{j}\left(n, \hat{b}_{\vec{\gamma}_{n, s-1}^{0}}\right) & G_{j}\left(n, \tilde{b}_{\vec{\gamma}_{n, s-1}^{0}}\right) & H_{j}\left(n, b_{\vec{\gamma}_{n, s-1}^{0}}\right)
\end{array}\right]
\end{array}
$$

by symmetry, so that

$$
\bar{B}_{j}\left(n, \vec{\gamma}_{n, s-1}^{0}\right)=B_{j}\left(n, \vec{\gamma}_{n, s-1}^{0}\right) E_{0}
$$

where

24) can be rewritten as

$$
E_{0}=\left[\begin{array}{ccccc}
1 & 0 & 0 & 0 & 0 \\
0 & 1 & 0 & 0 & 0 \\
0 & 0 & 0 & 1 & 0 \\
0 & 0 & 1 & 0 & 0 \\
0 & 0 & 0 & 0 & 1
\end{array}\right]
$$

$$
B_{j}\left(n+1, \vec{\gamma}_{n+1, s}\right)=B_{j}\left(n, \vec{\gamma}_{n, s-1}^{0}\right) E_{0} R
$$

if $\gamma_{3}=0$.

Let $E_{1}=I_{5 \times 5}$ be the identity matrix. [20], 23] and (25) can be combined to give

$$
B_{j}\left(n+1, \vec{\gamma}_{n+1, s}\right)=B_{j}\left(n, \vec{\gamma}_{n, s-1}^{\gamma_{3}}\right) E_{\gamma_{3}} R,
$$

where $\gamma_{3} \in\{0,1,2\}$. As $F_{j}\left(n+1, x_{\vec{\gamma}_{n+1, s}}\right)=F_{j}\left(n+1, \tilde{x}_{\vec{\gamma}_{n+1, s}}\right)$ for $x=a, b, c$, the first column of the matrix in 26) gives $F_{j}\left(n+1, x_{\vec{\gamma}_{n+1, s}}\right)$ for any vertex $x_{\vec{\gamma}_{n+1, s}}$ in terms of the quantities for $x_{\vec{\gamma}_{n, s-1}}$.

Proposition 5.2 For the Sierpinski gasket $S G(n+1)$ with $n \geq 2$, consider the vertex $x_{\vec{\gamma}_{n+1, s}}$ where $\vec{\gamma}_{n+1, s}=\left(n, 1, \gamma_{3}, \ldots, \gamma_{s}\right)$ with $3 \leq s \leq n+1$ and $\gamma_{k} \in\{0,1,2\}$ for $k \in\{3,4, . ., s\}$.

$$
\left[\begin{array}{c}
F_{j}\left(n+1, a_{\vec{\gamma}_{n+1, s}}\right) \\
F_{j}\left(n+1, b_{\vec{\gamma}_{n+1, s}}\right) \\
F_{j}\left(n+1, c_{\vec{\gamma}_{n+1, s}}\right)
\end{array}\right]=B_{j}\left(n, \vec{\gamma}_{n, s-1}^{\gamma_{3}}\right) E_{\gamma_{3}} R e_{1} .
$$

Using Theorems 3.1 4.1 and Propositions 5.1, 5.2 repeatedly, $F_{j}\left(n+1, x_{\vec{\gamma}_{n+1, s}}\right)$ for all the vertices of $S G(n+1)$ can be obtained.

\section{Summation and average of $F_{j}(n, x)$ over all the vertices of $S G(n)$}

It is worthwhile to derive the summation of $F_{j}(n, x)$ over all the vertices $x$ of $S G(n)$, defined as

$$
\Phi_{j}(n)=\sum_{x \in V(S G(n))} F_{j}(n, x),
$$

and the average of $F_{j}(n, x)$ over all the vertices, defined as

$$
\phi_{j}(n)=\frac{\Phi_{j}(n)}{v(S G(n))}=\frac{\Phi_{j}(n)}{\frac{3}{2}\left(3^{n}+1\right)} .
$$


It is clear that for any non-negative integer $n$,

$$
\sum_{j=1}^{4} \phi_{j}(n)=1
$$

For the vertices $x_{\vec{\gamma}_{n, s}}$ with $s=1$, i.e. $a_{m}, b_{m}$ and $c_{m}$, define their sum

$$
X_{j}(n, m)=F_{j}\left(n, a_{m}\right)+F_{j}\left(n, b_{m}\right)+F_{j}\left(n, c_{m}\right) .
$$

Similarly for the vertices with $s=2$, define

$$
Y_{j}\left(n, m^{\prime}\right)=F_{j}\left(n, a_{m^{\prime}, 1}\right)+F_{j}\left(n, b_{m^{\prime}, 1}\right)+F_{j}\left(n, c_{m^{\prime}, 1}\right),
$$

where $m \geq 0, m^{\prime} \geq 1$ and $n$ is larger than $m$ and $m^{\prime}$. By 15 , we have

$$
\begin{gathered}
X_{j}(n, m)=(1,1,1) B_{j}(n, m) e_{1}=(1,1,1) B_{j}(m+1, m) L^{n-m-1} e_{1}, \\
\begin{aligned}
Y_{j}\left(n, m^{\prime}\right) & =(1,1,1) B_{j}\left(n,\left(m^{\prime}, 1\right)\right) e_{1}=(1,1,1) B_{j}\left(m^{\prime}+1,\left(m^{\prime}, 1\right)\right) L^{n-m^{\prime}-1} e_{1} \\
& =(1,1,1) B_{j}\left(m^{\prime}, m^{\prime}-1\right) R L^{n-m^{\prime}-1} e_{1} .
\end{aligned}
\end{gathered}
$$

The first few $\Phi_{j}(n)$ are

$$
\begin{aligned}
& \Phi_{j}(0)=3 F_{j}(0, o), \\
& \Phi_{j}(1)=3 F_{j}(1, o)+X_{j}(1,0)=3 F_{j}(1, o)+2 F_{j}\left(1, a_{0}\right)+F_{j}\left(1, c_{0}\right),
\end{aligned}
$$

and

$$
\begin{aligned}
\Phi_{j}(2) & =3 F_{j}(2, o)+X_{j}(2,0)+X_{j}(2,1)+2 Y_{j}(2,1) \\
& =3 F_{j}(2, o)+(1,1,1)\left\{B_{j}(1,0) L+B_{j}(2,1)+2 B_{j}(1,0) R\right\} e_{1} .
\end{aligned}
$$

The corresponding values for $j \in\{1,2,3,4\}$ are

$$
\begin{array}{ll}
\Phi_{1}(0)=\frac{2}{3}, & \Phi_{2}(0)=\frac{1}{3}, \quad \Phi_{3}(0)=\Phi_{4}(0)=0, \\
\Phi_{1}(1)=\frac{1}{2}, & \Phi_{2}(1)=\frac{19}{54}, \quad \Phi_{3}(1)=\frac{7}{54}, \quad \Phi_{4}(1)=\frac{1}{54}, \\
\Phi_{1}(2)=\frac{163}{450}, & \Phi_{2}(2)=\frac{5257}{12150}, \quad \Phi_{3}(2)=\frac{2203}{12150}, \quad \Phi_{4}(2)=\frac{289}{12150} .
\end{array}
$$

For $n \geq 3$, we need the summation

$$
M_{j}(n)=\sum_{s=3}^{n}\left\{\sum_{\gamma_{s}=0}^{2} \sum_{\gamma_{s-1}=0}^{2} \cdots \sum_{\gamma_{3}=0}^{2} B_{j}\left(n, \vec{\gamma}_{n, s}\right)\right\}
$$


for the vertices $x_{\vec{\gamma}_{n, s}}$ with $s \geq 3$. By 18 and $26, M_{j}(3)=\sum_{\gamma_{3}=0}^{2} B_{j}\left(3, \vec{\gamma}_{3,3}\right)$ is given by

$$
\begin{aligned}
M_{j}(3) & =\left\{B_{j}\left(2, \vec{\gamma}_{2,2}^{0}\right) E_{0}+B_{j}\left(2, \vec{\gamma}_{2,2}^{1}\right) E_{1}+B_{j}\left(2, \vec{\gamma}_{2,2}^{2}\right) E_{2}\right\} R \\
& =B_{j}(2,(1,1))\left[E_{0}+E_{1}+E_{2}\right] R \\
& =B_{j}(1,0) R\left[E_{0}+E_{1}+E_{2}\right] R=B_{j}(1,0) R E R,
\end{aligned}
$$

where

$$
E=E_{0}+E_{1}+E_{2}=\left[\begin{array}{ccccc}
3 & 0 & 0 & 0 & 0 \\
0 & 2 & 1 & 0 & 0 \\
0 & 1 & 1 & 1 & 0 \\
0 & 0 & 1 & 2 & 0 \\
0 & 0 & 0 & 0 & 3
\end{array}\right]
$$

The general expression for $n \geq 3$ is

$$
\begin{aligned}
M_{j}( & n+1) \\
= & \sum_{s=3}^{n+1}\left\{\sum_{\gamma_{s}=0}^{2} \sum_{\gamma_{s-1}=0}^{2} \cdots \sum_{\gamma_{3}=0}^{2} B_{j}\left(n+1, \vec{\gamma}_{n+1, s}\right)\right\} \\
= & \left\{B_{j}\left(n, \vec{\gamma}_{n, 2}^{0}\right) E_{0}+B_{j}\left(n, \vec{\gamma}_{n, 2}^{1}\right) E_{1}+B_{j}\left(n, \vec{\gamma}_{n, 2}^{2}\right) E_{2}\right\} R \\
& +\sum_{s=4}^{n+1}\left\{\sum_{\gamma_{s}=0}^{2} \sum_{\gamma_{s-1}=0}^{2} \cdots \sum_{\gamma_{4}=0}^{2}\left[B_{j}\left(n, \vec{\gamma}_{n, s-1}^{0}\right) E_{0}+B_{j}\left(n, \vec{\gamma}_{n, s-1}^{1}\right) E_{1}+B_{j}\left(n, \vec{\gamma}_{n, s-1}^{2}\right) E_{2}\right] R\right\} \\
= & {\left[B_{j}(n,(n-1,1))+\sum_{s=3}^{n}\left\{\sum_{\gamma_{s}=0}^{2} \sum_{\gamma_{s-1}=0}^{2} \cdots \sum_{\gamma_{3}=0}^{2} B_{j}\left(n, \vec{\gamma}_{n, s}\right)\right\}\right] E R } \\
= & \left\{B_{j}(n-1, n-2) R+M_{j}(n)\right\} E R \\
= & B_{j}(n-1, n-2) R E R+B_{j}(n-2, n-3) R(E R)^{2}+M_{j}(n-1)(E R)^{2} \\
= & \cdots \\
= & \sum_{m=1}^{n-1} B_{j}(m, m-1) R(E R)^{n-m} .
\end{aligned}
$$

For example,

$$
\begin{aligned}
\Phi_{j}(3)= & 3 F_{j}(3, o)+X_{j}(3,0)+X_{j}(3,1)+X_{j}(3,2) \\
& +2\left[Y_{j}(3,1)+Y_{j}(3,2)+(1,1,1) B_{j}(1,0) R E R e_{1}\right] \\
= & 3 F_{j}(3, o)+(1,1,1)\left\{B_{j}(1,0) L^{2}+B_{j}(2,1) L+B_{j}(3,2)\right. \\
& \left.+2\left[B_{j}(1,0) R L+B_{j}(2,1) R+B_{j}(1,0) R E R\right]\right\} e_{1} \\
= & 3 F_{j}(3, o)+(1,1,1)\left\{B_{j}(1,0)\left[L^{2}+2 R L+2 R E R\right]+B_{j}(2,1)[L+2 R]+B_{j}(3,2)\right\} e_{1} .
\end{aligned}
$$

For general $n \geq 3$, we have

$$
\Phi_{j}(n)=3 F_{j}(n, o)+\sum_{m=0}^{n-1} X_{j}(n, m)+2 \sum_{m=1}^{n-1} Y_{j}(n, m)+2(1,1,1)\left\{\sum_{m=3}^{n} M_{j}(m) L^{n-m}\right\} e_{1},
$$


with $X_{j}(n, m)$ and $Y_{j}(n, m)$ given in 27) and 28), respectively. From (29), the summation in the last term of (30) is

$$
\begin{aligned}
\sum_{m=3}^{n} M_{j}(m) L^{n-m} & =\sum_{m=3}^{n}\left[\sum_{s=1}^{m-2} B_{j}(s, s-1) R(E R)^{m-1-s}\right] L^{n-m} \\
& =\sum_{m=2}^{n-1}\left[\sum_{s=1}^{m-1} B_{j}(s, s-1) R(E R)^{m-s}\right] L^{n-1-m} \\
& =\sum_{s=1}^{n-2} B_{j}(s, s-1) \sum_{m=s+1}^{n-1}\left[R(E R)^{m-s} L^{n-1-m}\right] \\
& =\sum_{s=1}^{n-2} B_{j}(s, s-1) \sum_{m=1}^{n-s-1}\left[R(E R)^{m} L^{n-1-m-s}\right]
\end{aligned}
$$

so that $\Phi_{j}(n)$ can be calculated exactly for any positive integer $n$.

Proposition 6.1 For the Sierpinski gasket $S G(n)$ with $n \geq 3$, the summation of $F_{j}(n, x)$ over all the vertex is given by

$$
\begin{aligned}
\Phi_{j}(n)= & (1,1,1)\left\{\sum_{m=1}^{n} B_{j}(m, m-1) L^{n-m}+2 \sum_{m=1}^{n-1} B_{j}(m, m-1) R L^{n-m-1}\right. \\
& \left.+2 \sum_{m=1}^{n-2} B_{j}(m, m-1) \sum_{s=1}^{n-m-1}\left[R(E R)^{s} L^{n-1-m-s}\right]\right\} e_{1}+3 F_{j}(n, o) \\
= & (1,1,1)\left\{\sum_{m=1}^{n} B_{j}(m, m-1) L^{n-m}+2 B_{j}(n-1, n-2) R\right. \\
& \left.+2 \sum_{m=1}^{n-2} B_{j}(m, m-1)\left[\sum_{s=0}^{n-m-1} R(E R)^{s} L^{n-m-1-s}\right]\right\} e_{1}+3 F_{j}(n, o) .
\end{aligned}
$$

Let us consider limiting distribution

$$
\lim _{n \rightarrow \infty} \phi_{j}(n) \equiv \phi_{j}
$$

with $j \in\{1,2,3,4\}$. It is easy to see that the term $3 F_{j}(n, o)$ in 31 can be neglected in the infinite $n$ limit for $\phi_{j}$, namely,

$$
\lim _{n \rightarrow \infty} \frac{F_{j}(n, o)}{\frac{3}{2}\left(3^{n}+1\right)}=0
$$

as the value $F_{j}(n, o)$ is between 0 and 1 for the four possible $j$. Similarly, the values of the quantities $F_{j}\left(m, x_{m-1}\right), G_{j}\left(m, x_{m-1}\right), H_{j}\left(m, x_{m-1}\right)$ with $x=a, b, c$ in the matrix $B_{j}(m, m-1)$ are between 0 and 1 , and all the eigenvalues of $R$ given in (17) are positive and less than or equal to 1 , such that

$$
\lim _{n \rightarrow \infty} \frac{(1,1,1)\left\{\sum_{m=1}^{n} B_{j}(m, m-1) L^{n-m}+2 B_{j}(n-1, n-2) R\right\} e_{1}}{\frac{3}{2}\left(3^{n}+1\right)} \leq \lim _{n \rightarrow \infty} \frac{3 n+6}{\frac{3}{2}\left(3^{n}+1\right)}=0 .
$$


Therefore, only the double summation term in (31) gives a non-zero contribution to $\phi_{j}$. Rewrite $E R=$ $Q_{1}\left[D_{1}+\bar{D}_{1}\right] Q_{1}^{-1}$ and $L=Q_{2} D_{2} Q_{2}^{-1}$, where

$$
Q_{1}=\left[\begin{array}{ccccc}
159 & -87 & 0 & -3 & -3 \\
38 & 14 & 1 & 2 & 1 \\
38 & 14 & 0 & 2 & -4 \\
38 & 14 & -1 & 2 & 1 \\
15 & 45 & 0 & -3 & 5
\end{array}\right], \quad Q_{2}=\left[\begin{array}{ccccc}
18 & 0 & -27 & 0 & -2 \\
5 & -1 & 98 & -1 & 1 \\
5 & 0 & -32 & 1 & 1 \\
0 & 1 & -52 & 0 & 0 \\
0 & 0 & 13 & 0 & 0
\end{array}\right]
$$

and

$$
D_{1}=\left[\begin{array}{ccccc}
0 & 0 & 0 & 0 & 0 \\
0 & 1 & 0 & 0 & 0 \\
0 & 0 & \frac{2}{5} & 0 & 0 \\
0 & 0 & 0 & \frac{3}{25} & 0 \\
0 & 0 & 0 & 0 & 0
\end{array}\right], \quad \bar{D}_{1}=\left[\begin{array}{ccccc}
3 & 0 & 0 & 0 & 0 \\
0 & 0 & 0 & 0 & 0 \\
0 & 0 & 0 & 0 & 0 \\
0 & 0 & 0 & 0 & 0 \\
0 & 0 & 0 & 0 & 0
\end{array}\right], \quad D_{2}=\left[\begin{array}{ccccc}
1 & 0 & 0 & 0 & 0 \\
0 & \frac{3}{10} & 0 & 0 & 0 \\
0 & 0 & \frac{6}{25} & 0 & 0 \\
0 & 0 & 0 & \frac{1}{5} & 0 \\
0 & 0 & 0 & 0 & \frac{1}{15}
\end{array}\right]
$$

then

$$
\begin{aligned}
\sum_{s=0}^{n-m-1} R(E R)^{s} L^{n-m-1-s}= & R \sum_{s=0}^{n-m-1}\left[Q_{1}\left(D_{1}^{s}+\bar{D}_{1}^{s}\right) Q_{1}^{-1}\right]\left[Q_{2} D_{2}^{n-m-1-s} Q_{2}^{-1}\right] \\
= & R \sum_{s=0}^{n-m-1}\left[Q_{1} D_{1}^{s} Q_{1}^{-1}\right]\left[Q_{2} D_{2}^{n-m-1-s} Q_{2}^{-1}\right] \\
& +R \sum_{s=0}^{n-m-1}\left[Q_{1} \bar{D}_{1}^{s} Q_{1}^{-1}\right]\left[Q_{2} D_{2}^{n-m-1-s} Q_{2}^{-1}\right]
\end{aligned}
$$

The elements of $B_{j}(m, m-1)$ have been solved in 36 - (39). Define

$$
\begin{aligned}
& Z_{j}(m)=(1,1,1) B_{j}(m, m-1) \\
& \quad=\left\{\begin{array}{l}
\lambda_{1}^{(1)}\left(\frac{3}{5}\right)^{m}+\lambda_{2}^{(1)}\left(\frac{1}{25}\right)^{m}+\lambda_{3}^{(1)}\left(\frac{1}{375}\right)^{m} \text { for } j=1, \\
\frac{363}{196} \lambda_{0}+\lambda_{1}^{(2)}\left(\frac{3}{5}\right)^{m}+\lambda_{2}^{(2)}\left(\frac{1}{25}\right)^{m}+\lambda_{3}^{(2)}\left(\frac{1}{375}\right)^{m}+\lambda_{4}^{(2)}\left(\frac{1}{15}\right)^{m}+\lambda_{5}^{(2)}\left(\frac{1}{225}\right)^{m} \text { for } j=2, \\
\frac{99}{98} \lambda_{0}+\lambda_{1}^{(3)}\left(\frac{3}{5}\right)^{m}+\lambda_{2}^{(3)}\left(\frac{1}{25}\right)^{m}+\lambda_{3}^{(3)}\left(\frac{1}{375}\right)^{m}+\lambda_{4}^{(3)}\left(\frac{1}{15}\right)^{m}+\lambda_{5}^{(3)}\left(\frac{1}{225}\right)^{m} \text { for } j=3, \\
\frac{27}{196} \lambda_{0}+\lambda_{1}^{(4)}\left(\frac{3}{5}\right)^{m}+\lambda_{2}^{(4)}\left(\frac{1}{25}\right)^{m}+\lambda_{3}^{(4)}\left(\frac{1}{375}\right)^{m}+\lambda_{4}^{(4)}\left(\frac{1}{15}\right)^{m}+\lambda_{5}^{(4)}\left(\frac{1}{225}\right)^{m} \text { for } j=4,
\end{array}\right.
\end{aligned}
$$

where $\lambda_{0}=(1,1,1,1,1)$ and

$$
\begin{aligned}
& \lambda_{1}^{(1)}=\left(\frac{605}{392}, \frac{121}{56}, \frac{121}{56}, \frac{121}{56}, \frac{1089}{392}\right), \quad \lambda_{2}^{(1)}=\left(\frac{-1375}{196}, \frac{-55}{28}, \frac{-55}{28}, \frac{-55}{28}, \frac{1221}{196}\right), \\
& \lambda_{3}^{(1)}=\left(\frac{3125}{392}, \frac{-375}{56}, \frac{-375}{56}, \frac{-375}{56}, \frac{585}{392}\right), \quad \lambda_{1}^{(2)}=\left(\frac{-275}{396}, \frac{-55}{56}, \frac{-55}{56}, \frac{-55}{56}, \frac{-495}{392}\right), \\
& \lambda_{2}^{(2)}=\left(\frac{2375}{196}, \frac{95}{28}, \frac{95}{28}, \frac{95}{28}, \frac{-2109}{196}\right), \quad \lambda_{3}^{(2)}=\left(\frac{-9375}{392}, \frac{1125}{56}, \frac{1125}{56}, \frac{1125}{56}, \frac{-1755}{392}\right), \\
& \lambda_{4}^{(2)}=\left(\frac{-1265}{196}, \frac{-187}{196}, \frac{-187}{196}, \frac{-187}{196}, \frac{891}{196}\right), \quad \lambda_{5}^{(2)}=\left(\frac{10}{49}, \frac{-240}{49}, \frac{-240}{49}, \frac{-240}{49}, \frac{54}{49}\right),
\end{aligned}
$$




$$
\begin{aligned}
& \lambda_{1}^{(3)}=\left(\frac{-285}{392}, \frac{-57}{56}, \frac{-57}{56}, \frac{-57}{56}, \frac{-513}{392}\right), \quad \lambda_{2}^{(3)}=\left(\frac{-625}{196}, \frac{-25}{28}, \frac{-25}{28}, \frac{-25}{28}, \frac{555}{196}\right), \\
& \lambda_{3}^{(3)}=\left(\frac{-9375}{392}, \frac{-1125}{56}, \frac{-1125}{56}, \frac{-1125}{56}, \frac{1755}{392}\right), \quad \lambda_{4}^{(3)}=\left(\frac{230}{49}, \frac{34}{49}, \frac{34}{49}, \frac{34}{49}, \frac{-162}{49}\right), \\
& \lambda_{5}^{(3)}=\left(\frac{500}{49}, \frac{480}{49}, \frac{480}{49}, \frac{480}{49}, \frac{-108}{49}\right), \quad \lambda_{1}^{(4)}=\left(\frac{-45}{392}, \frac{-9}{56}, \frac{-9}{56}, \frac{-9}{56}, \frac{-81}{392}\right), \\
& \lambda_{2}^{(4)}=\left(\frac{-375}{196}, \frac{-15}{28}, \frac{-15}{28}, \frac{-15}{28}, \frac{333}{196}\right), \quad \lambda_{3}^{(4)}=\left(\frac{-3125}{392}, \frac{375}{56}, \frac{375}{56}, \frac{375}{56}, \frac{-585}{392}\right), \\
& \lambda_{4}^{(4)}=\left(\frac{345}{196}, \frac{51}{196}, \frac{51}{196}, \frac{51}{196}, \frac{-243}{196}\right), \quad \lambda_{5}^{(4)}=\left(\frac{250}{49}, \frac{-240}{49}, \frac{-240}{49}, \frac{-240}{49}, \frac{54}{49}\right) .
\end{aligned}
$$

Substituting 32) into 31, we get

$$
\begin{aligned}
\phi_{j}= & \lim _{n \rightarrow \infty} \frac{4}{3}\left(3^{n}+1\right)^{-1} \sum_{m=1}^{n-2} Z_{j}(m) R\left\{\sum_{s=0}^{n-m-1}\left[Q_{1} D_{1}^{s} Q_{1}^{-1}\right]\left[Q_{2} D_{2}^{n-m-1-s} Q_{2}^{-1}\right]\right. \\
& \left.+\sum_{s=0}^{n-m-1}\left[Q_{1} \bar{D}_{1}^{s} Q_{1}^{-1}\right]\left[Q_{2} D_{2}^{n-m-1-s} Q_{2}^{-1}\right]\right\} e_{1} .
\end{aligned}
$$

As the eigenvalues of $D_{1}$ and $D_{2}$ are between 0 and 1, the first term in (34) makes no contributions since

$$
\begin{aligned}
0 & <\sum_{m=1}^{n-2} Z_{j}(m) R \sum_{s=0}^{n-m-1}\left[Q_{1} D_{1}^{s} Q_{1}^{-1}\right]\left[Q_{2} D_{2}^{n-m-1-s} Q_{2}^{-1}\right] e_{1} \\
& \leq \sum_{m=1}^{n-2} Z_{j}(m) R \sum_{s=0}^{n-m-1}\left[Q_{1} I Q_{1}^{-1}\right]\left[Q_{2} I Q_{2}^{-1}\right] e_{1} \leq 3 n^{2} .
\end{aligned}
$$

Consider the second term in 34,

$$
\begin{aligned}
& \sum_{m=1}^{n-2} Z_{j}(m) R \sum_{s=0}^{n-m-1}\left[Q_{1} \bar{D}_{1}^{s} Q_{1}^{-1}\right]\left[Q_{2} D_{2}^{n-m-1-s} Q_{2}^{-1}\right] \\
= & \sum_{m=1}^{n-2} Z_{j}(m) R\left[Q_{1} \tilde{D}_{1} Q_{1}^{-1}\right] \sum_{s=0}^{n-m-1} 3^{s}\left[Q_{2} D_{2}^{n-m-1-s} Q_{2}^{-1}\right] \\
= & \sum_{m=1}^{n-2} Z_{j}(m) R\left[Q_{1} \tilde{D}_{1} Q_{1}^{-1}\right] Q_{2} D(n, m) Q_{2}^{-1},
\end{aligned}
$$

where

$$
\tilde{D}_{1}=\left[\begin{array}{ccccc}
1 & 0 & 0 & 0 & 0 \\
0 & 0 & 0 & 0 & 0 \\
0 & 0 & 0 & 0 & 0 \\
0 & 0 & 0 & 0 & 0 \\
0 & 0 & 0 & 0 & 0
\end{array}\right]
$$

and define

$$
D(n, m) \equiv \sum_{s=0}^{n-m-1} 3^{s} D_{2}^{n-m-1-s}
$$




$$
\begin{aligned}
& =\sum_{s=0}^{n-m-1}\left[\begin{array}{ccccc}
3^{s}\left(\frac{6}{25}\right)^{n-m-1-s} & 0 & 0 & 0 & 0 \\
0 & 3^{s}\left(\frac{1}{15}\right)^{n-m-1-s} & 0 & 0 & 0 \\
0 & 0 & 3^{s} & 0 & 0 \\
0 & 0 & 0 & 3^{s}\left(\frac{1}{5}\right)^{n-m-1-s} & 0 \\
0 & 0 & 0 & 0 & 3^{s}\left(\frac{3}{10}\right)^{n-m-1-s}
\end{array}\right] \\
& =\left[\begin{array}{ccccc}
\frac{25\left[3^{n-m}-\left(\frac{6}{25}\right)^{n-m}\right]}{69} & 0 & 0 & 0 & 0 \\
0 & \frac{15\left[3^{n-m}-\left(\frac{1}{15}\right)^{n-m}\right]}{44} & 0 & 0 & 0 \\
0 & 0 & \frac{3^{n-m}-1}{2} & 0 & 0 \\
0 & 0 & 0 & \frac{5\left[3^{n-m}-\left(\frac{1}{5}\right)^{n-m}\right]}{14} & \frac{10\left[3^{n-m}-\left(\frac{3}{10}\right)^{n-m}\right]}{27}
\end{array}\right] \\
& \equiv 3^{n-m} \mathcal{D}+\mathcal{D}_{2}(n-m), \\
& 0
\end{aligned}
$$

with

$$
\begin{aligned}
& \mathcal{D}=\left[\begin{array}{ccccc}
\frac{25}{69} & 0 & 0 & 0 & 0 \\
0 & \frac{15}{44} & 0 & 0 & 0 \\
0 & 0 & \frac{1}{2} & 0 & 0 \\
0 & 0 & 0 & \frac{5}{14} & 0 \\
0 & 0 & 0 & 0 & \frac{10}{27}
\end{array}\right] \\
& \mathcal{D}_{2}(n-m)=\left[\begin{array}{ccccc}
\frac{-25\left(\frac{6}{25}\right)^{n-m}}{69} & 0 & 0 & 0 & 0 \\
0 & \frac{-\left(\frac{1}{15}\right)^{n-m-1}}{44} & 0 & 0 & 0 \\
0 & 0 & \frac{-1}{2} & 0 & 0 \\
0 & 0 & 0 & \frac{-\left(\frac{1}{5}\right)^{n-m-1}}{14} & 0 \\
0 & 0 & 0 & 0 & \frac{-\left(\frac{3}{10}\right)^{n-m-1}}{9}
\end{array}\right] \text {. }
\end{aligned}
$$

Since the absolute values of all the eigenvalues of $\mathcal{D}_{2}(n-m)$ are less than one, we have

$$
\begin{aligned}
\phi_{j}= & \lim _{n \rightarrow \infty} \frac{4}{3}\left(3^{n}+1\right)^{-1} \sum_{m=1}^{n-2}\left\{3^{n-m} Z_{j}(m) R Q_{1} \tilde{D}_{1} Q_{1}^{-1} Q_{2} \mathcal{D} Q_{2}^{-1}\right. \\
& \left.+Z_{j}(m) R Q_{1} \tilde{D}_{1} Q_{1}^{-1} Q_{2} \mathcal{D}_{2}(n-m) Q_{2}^{-1}\right\} e_{1} \\
= & \lim _{n \rightarrow \infty} \frac{4}{3}\left(3^{n}+1\right)^{-1} \sum_{m=1}^{n-2} 3^{n-m} Z_{j}(m) \tilde{R} e_{1},
\end{aligned}
$$

where $\tilde{R}=R Q_{1} \tilde{D}_{1} Q_{1}^{-1} Q_{2} \mathcal{D} Q_{2}^{-1}$. Substituting the expression of $Z_{j}(m)$ from 33 into 35 , carrying out the summation and taking the infinite $n$ limit, we arrive at

$$
\phi_{j}=\frac{4}{3} \times\left\{\begin{array}{l}
{\left[\frac{\lambda_{1}^{(1)}}{4}+\frac{\lambda_{2}^{(1)}}{74}+\frac{\lambda_{3}^{(1)}}{1124}\right] \tilde{R} e_{1} \text { for } j=1,} \\
{\left[\frac{363 \lambda_{0}}{392}+\frac{\lambda_{1}^{(2)}}{4}+\frac{\lambda_{2}^{(2)}}{74}+\frac{\lambda_{3}^{(2)}}{1124}+\frac{\lambda_{4}^{(2)}}{44}+\frac{\lambda_{5}^{(2)}}{674}\right] \tilde{R} e_{1} \text { for } j=2,} \\
{\left[\frac{99 \lambda_{0}}{196}+\frac{\lambda_{1}^{(3)}}{4}+\frac{\lambda_{2}^{(3)}}{74}+\frac{\lambda_{3}^{(3)}}{1124}+\frac{\lambda_{4}^{(3)}}{44}+\frac{\lambda_{5}^{(3)}}{674}\right] \tilde{R} e_{1} \text { for } j=3,} \\
{\left[\frac{27 \lambda_{0}}{392}+\frac{\lambda_{1}^{(4)}}{4}+\frac{\lambda_{2}^{(4)}}{74}+\frac{\lambda_{3}^{(4)}}{1124}+\frac{\lambda_{4}^{(4)}}{44}+\frac{\lambda_{5}^{(4)}}{674}\right] \tilde{R} e_{1} \text { for } j=4 .}
\end{array}\right.
$$


Tab. 1: Numerical values of $\phi_{j}(n)$ with $j \in\{1,2,3,4\}$, and the comparison of $\phi_{j}$ with $f_{j}$ for the square lattice $(s q)$. The last digits given are rounded off.

\begin{tabular}{|c|c|c|c|c|}
\hline$n$ & $\phi_{1}(n)$ & $\phi_{2}(n)$ & $\phi_{3}(n)$ & $\phi_{4}(n)$ \\
\hline$\overline{0} 0$ & $\begin{array}{c}\frac{2}{3} \\
=0.6666666667\end{array}$ & $\begin{array}{c}\frac{1}{3} \\
=0.3333333333\end{array}$ & 0 & 0 \\
\hline 1 & $\begin{aligned} & \frac{1}{2} \\
= & 0.5\end{aligned}$ & $\begin{array}{c}\frac{19}{54} \\
=0.3518518519\end{array}$ & $\begin{array}{c}\frac{7}{54} \\
=0.1296296296\end{array}$ & $=0.01851851852$ \\
\hline 2 & $\begin{array}{c}\frac{163}{450} \\
=0.3622222222\end{array}$ & $\begin{array}{c}\frac{5257}{12150} \\
=0.4326748971\end{array}$ & $\begin{array}{c}\frac{2203}{12150} \\
=0.1813168724\end{array}$ & $\begin{array}{c}\frac{289}{12150} \\
=0.02378600823\end{array}$ \\
\hline 3 & $\begin{array}{c}\frac{143357}{472500} \\
=0.3034010582\end{array}$ & $\begin{array}{c}\frac{17871899}{38272500} \\
=0.4669645045\end{array}$ & $\begin{array}{c}\frac{7787951}{38272500} \\
=0.2034868639\end{array}$ & $\begin{array}{c}\frac{1000733}{38272500} \\
=0.02614757332\end{array}$ \\
\hline 4 & $\begin{array}{c}\frac{24381607}{86484375} \\
=0.2819192137\end{array}$ & $\begin{aligned} & \frac{30227565716}{63047109375} \\
= & 0.4794441175\end{aligned}$ & $\begin{aligned} & \frac{13341669059}{63047109375} \\
= & 0.2116142864\end{aligned}$ & $\begin{array}{c}\frac{1703683097}{63047109375} \\
=0.02702238237\end{array}$ \\
\hline 5 & $\begin{aligned} & \frac{39739246273}{144755859375} \\
= & 0.2745259946\end{aligned}$ & $\begin{array}{l}\frac{51047283737324}{105527021484375} \\
=0.4837366110\end{array}$ & $\begin{array}{l}\frac{22626394285676}{105527021484375} \\
=0.2144132751\end{array}$ & $\begin{aligned} & \frac{2883432928358}{105527021484375} \\
= & 0.02732411934\end{aligned}$ \\
\hline$\infty$ & $\begin{array}{c}\frac{10957}{40464} \\
=0.2707839067\end{array}$ & $\begin{array}{c}\frac{6626035}{13636368} \\
=0.4859090778\end{array}$ & $\begin{array}{c}\frac{2943139}{13636368} \\
=0.2158301243\end{array}$ & $\begin{array}{c}\frac{124895}{4545456} \\
=0.02747689121\end{array}$ \\
\hline$s q$ & $\overline{\bar{f} f_{1}}$ & $\overline{f_{2}}$ & $\overline{f_{3}}$ & $\overline{f_{4}}$ \\
\hline & $\begin{array}{l}\frac{8}{\pi^{2}}-\frac{16}{\pi^{3}} \\
=0.2945449182\end{array}$ & $\begin{aligned} & \frac{8}{\pi}-\frac{36}{\pi^{2}}+\frac{48}{\pi^{3}} \\
= & 0.4469901311\end{aligned}$ & $\begin{array}{l}2-\frac{16}{\pi}+\frac{48}{\pi^{2}}-\frac{48}{\pi^{3}} \\
=0.2223849831\end{array}$ & $\begin{array}{c}-1+\frac{8}{\pi}-\frac{20}{\pi^{2}}+\frac{16}{\pi^{3}} \\
=0.03607996755\end{array}$ \\
\hline
\end{tabular}

The matrix productions can be done to give the following theorem.

Theorem 6.1 Consider all the vertices of the Sierpinski gasket $S G(n)$ in the infinite $n$ limit. The average probabilities that a vertex is connected by 1,2, 3 or 4 bond(s) among all the spanning tree configurations are

$$
\begin{aligned}
& \phi_{1}=\frac{10957}{40464}=0.270783906682 \cdots, \quad \phi_{2}=\frac{6626035}{13636368}=0.485909077842 \cdots, \\
& \phi_{3}=\frac{2943139}{13636368}=0.215830124267 \cdots, \quad \phi_{4}=\frac{124895}{4545456}=0.0274768912073 \cdots .
\end{aligned}
$$

For the Sierpinski gasket $S G(n)$ in the infinite $n$ limit, the average number of bonds connecting to a vertex among all the spanning tree configurations is equal to $\phi_{1}+2 \phi_{2}+3 \phi_{3}+4 \phi_{4}=2$ as expected [Aldous(1990)]. We list the numerical values of $\phi_{j}(n)$ with $j \in\{1,2,3,4\}$ for $0 \leq n \leq 5$ and infinite $n$ limit in Table 1. We find that $\phi_{1}(n)$ decreases monotonically as $n$ increases, while $\phi_{2}(n), \phi_{3}(n)$ and $\phi_{4}(n)$ increase monotonically. The values for $n=5$ are already very close to $\phi_{j}$ in the infinite $n$ limit with deviations about $1 \%$.

It is interesting to compare the Sierpinski gasket $S G(n)$ in the infinite $n$ limit with the infinite twodimensional square lattice which is also a 4-regular lattice. For the square lattice, all the vertices are identical due to the translational invariant, and the probabilities that a vertex is connected by 1, 2, 3 or 4 bond(s) among all the spanning tree configurations have been determined exactly in [Manna et al.(1992)]. 
They are denoted by $f_{j}$ with $j \in\{1,2,3,4\}$. As shown in Table 1 , $f_{1}, f_{3}$ and $f_{4}$ are slightly larger than $\phi_{1}, \phi_{3}, \phi_{4}$, respectively, while $f_{2}$ is smaller than $\phi_{2}$.

\section{Acknowledgements}

We would like to thank Akira Sakai for valuable comments and the anonymous referees for positive suggestions. The research of S.C.C. was partially supported by the NSC grants NSC-96-2112-M-006-001 and 97-2112-M-006-007-MY3. The research of L.C.C was partially supported by TJ\&MY Foundation and the NSC grant NSC-96-2115-M-030-002. L.C.C. would like to thank PIMS, university of British Columbia for the hospitality.

$$
\begin{array}{ll}
\text { A Recursion relations for } F_{j}\left(n+1, a_{n}\right), G_{j}\left(n+1, a_{n}\right), G_{j}\left(n+1, b_{n}\right), \\
& H_{j}\left(n+1, a_{n}\right) \text { with } j \in\{1,2,3,4\}
\end{array}
$$

Using Figs. $3 \mid 5$ for the vertex $a_{n}$ or $b_{n}$, we obtain the following recursion relations

$$
\begin{aligned}
& \left\{\begin{aligned}
f_{1}\left(n+1, a_{n}\right)= & 2 f_{1}(n, o) g_{0}\left(n, b_{n}\right) f(n) \\
f_{2}\left(n+1, a_{n}\right)= & 2 f_{2}(n, o) g_{0}\left(n, b_{n}\right) f(n)+2 f_{1}(n, o)\left[g_{1}(n, o)+g_{1}\left(n, b_{n}\right)\right] f(n) \\
& +2 f_{1}(n, o)^{2} g(n) \\
f_{3}\left(n+1, a_{n}\right)= & 2 f_{2}(n, o)\left[g_{1}(n, o)+g_{1}\left(n, b_{n}\right)\right] f(n)+4 f_{1}(n, o) f_{2}(n, o) g(n) \\
& +2 f_{1}(n, o)\left[g_{2}(n, o)+g_{2}\left(n, b_{n}\right)\right] f(n) \\
f_{4}\left(n+1, a_{n}\right)= & 2 f_{2}(n, o)\left[g_{2}(n, o)+g_{2}\left(n, b_{n}\right)\right] f(n)+2 f_{2}(n, o)^{2} g(n),
\end{aligned}\right. \\
& \left\{\begin{aligned}
g_{1}\left(n+1, a_{n}\right)= & 2 f_{1}(n, o) g_{0}\left(n, b_{n}\right) g(n) \\
g_{2}\left(n+1, a_{n}\right)= & f_{1}(n, o)^{2} h(n)+g_{1}(n, o)^{2} f(n)+4 f_{1}(n, o) g_{1}(n, o) g(n) \\
& +2\left[f_{1}(n) g_{1}\left(n, b_{n}\right)+f_{2}(n) g_{0}\left(n, b_{n}\right)\right] g(n), \\
g_{3}\left(n+1, a_{n}\right)= & 2 f_{2}(n, o) f_{1}(n, o) h(n)+2 g_{1}(n, o) g_{2}(n, o) f(n) \\
& +4\left[f_{2}(n, o) g_{1}(n, o)+f_{1}(n, o) g_{2}(n, o)\right] g(n) \\
& +2\left[f_{2}(n, o) g_{1}\left(n, b_{n}\right)+f_{1}(n, o) g_{2}\left(n, b_{n}\right)\right] g(n), \\
g_{4}\left(n+1, a_{n}\right)= & f_{2}(n, o)^{2} h(n)+f_{2}(n, o)\left[2 g_{2}\left(n, b_{n}\right)+4 g_{2}(n, o)\right] g(n)+g_{2}(n, o)^{2} f(n),
\end{aligned}\right. \\
& \left\{\begin{aligned}
g_{1}\left(n+1, b_{n}\right)= & f_{1}(n, o) h_{0}(n, o) f(n)+2 f_{1}(n, o) g_{0}\left(n, b_{n}\right) g(n) \\
& +2 g_{1}(n, o) g_{0}\left(n, b_{n}\right) f(n), \\
g_{2}\left(n+1, b_{n}\right)= & {\left[f_{2}(n, o) h_{0}(n, o)+f_{1}(n, o) h_{1}(n, o)\right] f(n)+2 f_{1}(n, o) g_{1}(n, o) g(n) } \\
& +g_{1}(n, o)^{2} f(n)+2\left[f_{2}(n, o) g_{0}\left(n, b_{n}\right)+f_{1}(n, o) g_{1}\left(n, b_{n}\right)\right] g(n) \\
& +2\left[g_{2}(n, o) g_{0}\left(n, b_{n}\right)+g_{1}(n, o) g_{1}\left(n, b_{n}\right)\right] f(n), \\
g_{3}\left(n+1, b_{n}\right)= & {\left[f_{2}(n, o) h_{1}(n, o)+f_{1}(n, o) h_{2}(n, o)\right] f(n) } \\
& +2\left[f_{2}(n, o) g_{1}(n, o)+f_{1}(n, o) g_{2}(n, o)\right] g(n) \\
& +2\left[f_{2}(n, o) g_{1}\left(n, b_{n}\right)+f_{1}(n, o) g_{2}\left(n, b_{n}\right)\right] g(n) \\
& +2\left[g_{2}(n, o) g_{1}\left(n, b_{n}\right)+g_{1}(n, o) g_{2}\left(n, b_{n}\right)\right] f(n)+2 g_{1}(n, o) g_{2}(n, o) f(n), \\
g_{4}\left(n+1, b_{n}\right)= & f_{2}(n, o) h_{2}(n, o) f(n)+2 g_{2}(n, o) g_{2}\left(n, b_{n}\right) f(n) \\
& +2 f_{2}(n, o)\left[g_{2}(n, o)+g_{2}\left(n, b_{n}\right)\right] g(n)+g_{2}(n, o)^{2} f(n),
\end{aligned}\right.
\end{aligned}
$$


and

$$
\left\{\begin{aligned}
h_{1}\left(n+1, a_{n}\right)= & 4 f_{1}(n, o) h_{0}(n, o) g(n)+2 f_{1}(n, o) g_{0}\left(n, b_{n}\right) h(n) \\
& +4 g_{1}(n, o) h_{0}(n, o) f(n)+8 g_{1}(n, o) g_{0}\left(n, b_{n}\right) g(n), \\
h_{2}\left(n+1, a_{n}\right)= & 4\left[f_{2}(n, o) h_{0}(n, o)+f_{1}(n, o) h_{1}(n, o)\right] g(n) \\
& +2\left[f_{1}(n, o) g_{1}\left(n, b_{n}\right)+f_{2}(n, o) g_{0}\left(n, b_{n}\right)\right] h(n) \\
& +4\left[g_{2}(n, o) h_{0}(n, o)+g_{1}(n, o) h_{1}(n, o)\right] f(n)+2 f_{1}(n, o) g_{1}(n, o) h(n) \\
& +6 g_{1}(n, o)^{2} g(n)+8\left[g_{2}(n, o) g_{0}\left(n, b_{n}\right)+g_{1}(n, o) g_{1}\left(n, b_{n}\right)\right] g(n), \\
h_{3}\left(n+1, a_{n}\right)= & 4\left[f_{2}(n, o) h_{1}(n, o)+f_{1}(n, o) h_{2}(n, o)\right] g(n) \\
& +2\left[f_{2}(n, o) g_{1}\left(n, b_{n}\right)+f_{1}(n, o) g_{2}\left(n, b_{n}\right)\right] h(n) \\
& +4\left[g_{2}(n, o) h_{1}(n, o)+g_{1}(n, o) h_{2}(n, o)\right] f(n) \\
& +2\left[f_{2}(n, o) g_{1}(n, o)+f_{1}(n, o) g_{2}(n, o)\right] h(n) \\
& +8\left[g_{2}(n, o) g_{1}\left(n, b_{n}\right)+g_{1}(n, o) g_{2}\left(n, b_{n}\right)\right] g(n) \\
& +12 g_{1}(n, o) g_{2}(n, o) g(n), \\
& 4 f_{2}(n, o) h_{2}(n, o) g(n)+2 f_{2}(n, o)\left[g_{2}\left(n, b_{n}\right)+g_{2}(n, o)\right] h(n) \\
& +4 g_{2}(n, o) h_{2}(n, o) f(n)+8 g_{2}(n, o) g_{2}\left(n, b_{n}\right) g(n)+6 g_{2}(n, o)^{2} g(n) .
\end{aligned}\right.
$$

Using the identity $3 g(n)^{2}=f(n) h(n)$, it follows that

$$
\begin{aligned}
& \left\{\begin{array}{l}
F_{1}\left(n+1, a_{n}\right)=\frac{F_{1}(n, o) G_{0}\left(n, b_{n}\right)}{3}, \\
F_{2}\left(n+1, a_{n}\right)=\frac{F_{2}(n, o) G_{0}\left(n, b_{n}\right)}{3}+\frac{F_{1}(n, o)^{2}}{3}+\frac{F_{1}(n, o)\left[G_{1}(n, o)+G_{1}\left(n, b_{n}\right)\right]}{3}, \\
F_{3}\left(n+1, a_{n}\right)=\frac{F_{2}(n, o)\left[G_{1}(n, o)+G_{1}\left(n, b_{n}\right)\right]}{3}+\frac{2 F_{1}(n, o) F_{2}(n, o)}{3}+\frac{F_{1}(n, o)\left[G_{2}(n, o)+G_{2}\left(n, b_{n}\right)\right]}{3}, \\
F_{4}\left(n+1, a_{n}\right)=\frac{F_{2}(n, o)\left[G_{2}(n, o)+G_{2}\left(n, b_{n}\right)\right]}{3}+\frac{F_{2}(n, o)^{2}}{3},
\end{array}\right. \\
& \left\{\begin{aligned}
G_{1}\left(n+1, a_{n}\right)= & \frac{F_{1}(n, o) G_{0}\left(n, b_{n}\right)}{5}, \\
G_{2}\left(n+1, a_{n}\right)= & \frac{3 F_{1}(n, o)^{2}}{10}+\frac{F_{1}(n, o)\left[2 G_{1}(n, o)+G_{1}\left(n, b_{n}\right)\right]}{5}+\frac{F_{2}(n, o) G_{0}\left(n, b_{n}\right)}{5}+\frac{G_{1}(n, o)^{2}}{10}, \\
G_{3}\left(n+1, a_{n}\right)= & \frac{3 F_{2}(n, o) F_{1}(n, o)}{5}+\frac{F_{2}(n, o)\left[2 G_{1}(n, o)+G_{1}\left(n, b_{n}\right)\right]}{5}+\frac{G_{2}(n, o) G_{1}(n, o)}{5} \\
& +\frac{F_{1}(n, o)\left[2 G_{2}(n, o)+G_{2}\left(n, b_{n}\right)\right]}{5}, \\
G_{4}\left(n+1, a_{n}\right)= & \frac{3 F_{2}(n, o)^{2}}{10}+\frac{F_{2}(n, o)\left[2 G_{2}(n, o)+G_{2}\left(n, b_{n}\right)\right]}{5}+\frac{G_{2}(n, o)^{2}}{10},
\end{aligned}\right. \\
& \left\{\begin{aligned}
G_{1}\left(n+1, b_{n}\right)= & \frac{3 F_{1}(n, o) H_{0}(n, o)}{10}+\frac{\left[F_{1}(n, o)+G_{1}(n, o)\right] G_{0}\left(n, b_{n}\right)}{5}, \\
G_{2}\left(n+1, b_{n}\right)= & \frac{3\left[F_{2}(n, o) H_{0}(n, o)+F_{1}(n, o) H_{1}(n, o)\right]}{10}+\frac{G_{1}(n, o)^{2}}{10}+\frac{F_{1}(n, o)\left[G_{1}(n, o)+G_{1}\left(n, b_{n}\right)\right]}{5} \\
& +\frac{F_{2}(n, o) G_{0}\left(n, b_{n}\right)}{5}+\frac{G_{0}\left(n, b_{n}\right) G_{2}(n, o)+G_{1}(n, o) G_{1}\left(n, b_{n}\right)}{5}, \\
G_{3}\left(n+1, b_{n}\right)= & \frac{3\left[F_{2}(n, o) H_{1}(n, o)+F_{1}(n, o) H_{2}(n, o)\right]}{10} \\
& +\frac{\left[G_{1}\left(n, b_{n}\right)+G_{1}(n, o)\right] G_{2}(n, o)+G_{1}(n, o) G_{2}\left(n, b_{n}\right)}{5} \\
& +\sum_{r=1}^{2} \frac{F_{r}(n, o)\left[G_{3-r}(n, o)+G_{3-r}\left(n, b_{n}\right)\right]}{5}, \\
G_{4}\left(n+1, b_{n}\right)= & \frac{3 F_{2}(n, o) H_{2}(n, o)}{10}+\frac{F_{2}(n, o)\left[G_{2}(n, o)+G_{2}\left(n, b_{n}\right)\right]+G_{2}(n, o) G_{2}\left(n, b_{n}\right)}{5}+\frac{G_{2}(n, o)^{2}}{10},
\end{aligned}\right.
\end{aligned}
$$


and

$$
\left\{\begin{aligned}
H_{1}\left(n+1, a_{n}\right)= & \frac{3 F_{1}(n, o)\left[2 H_{0}(n, o)+G_{0}\left(n, b_{n}\right)\right]}{25}+\frac{6 G_{1}(n, o) H_{0}(n, o)}{25}+\frac{4 G_{1}(n, o) G_{0}\left(n, b_{n}\right)}{25}, \\
H_{2}\left(n+1, a_{n}\right)= & \frac{6\left[F_{2}(n, o) H_{0}(n, o)+F_{1}(n, o) H_{1}(n, o)\right]}{25}+\frac{3 F_{1}(n, o)\left[G_{1}(n, o)+G_{1}\left(n, b_{n}\right)\right]}{25} \\
& +\frac{3 F_{2}(n, o) G_{0}\left(n, b_{n}\right)}{25}+\frac{4\left[G_{2}(n, o) G_{0}\left(n, b_{n}\right)+G_{1}(n, o) G_{1}\left(n, b_{n}\right)\right]}{25} \\
& +\frac{3 G_{1}(n, o)^{2}}{25}+\frac{6\left[H_{0}(n, o) G_{2}(n, o)+H_{1}(n, o) G_{1}(n, o)\right]}{25}, \\
H_{3}\left(n+1, a_{n}\right)= & \frac{6\left[F_{2}(n, o) H_{1}(n, o)+F_{1}(n, o) H_{2}(n, o)\right]}{25}+\frac{3 F_{2}(n, o)\left[G_{1}(n, o)+G_{1}\left(n, b_{n}\right)\right]}{25} \\
& +\frac{3 F_{1}(n, o)\left[G_{2}(n, o)+G_{2}\left(n, b_{n}\right)\right]}{25}+\frac{6\left[G_{2}(n, o) H_{1}(n, o)+G_{1}(n, o) H_{2}(n, o)\right]}{25} \\
& +\frac{\left[4 G_{1}\left(n, b_{n}\right)+6 G_{1}(n, o)\right] G_{2}(n, o)}{25}+\frac{4 G_{1}(n, o) G_{2}\left(n, b_{n}\right)}{25}, \\
H_{4}\left(n+1, a_{n}\right)= & \frac{6 F_{2}(n, o) H_{2}(n, o)}{25}+\frac{3 F_{2}(n, o)\left[G_{2}(n, o)+G_{2}\left(n, b_{n}\right)\right]}{25}+\frac{6 G_{2}(n, o) H_{2}(n, o)}{25} \\
& +\frac{4 G_{2}(n, o) G_{2}\left(n, b_{n}\right)}{25}+\frac{3 G_{2}(n, o)^{2}}{25} .
\end{aligned}\right.
$$

\section{References}

[Kirchhoff(1847)] G. Kirchhoff, Über die Auflösung der Gleichungen, auf welche man bei der Untersuchung der linearen Verteilung galvanischer Ströme geführt wird. Ann. Phys. Chem. 72: 497-508, 1847.

[Biggs(1993)] N. L. Biggs, Algebraic Graph Theory. 2nd ed. Cambridge University Press, Cambridge, 1993.

[Burton and Pemantle(1993)] R. Burton and R. Pemantle, Local characteristics, entropy and limit theorems for spanning trees and domino tilings via transfer-impedances. Ann. Probab. 21: 1329-1371, 1993.

[Lyons(2005)] R. Lyons, Asymptotic enumeration of spanning trees. Combin. Probab. Comput. 14: 491522, 2005.

[Welsh(1993)] D. J. A. Welsh, Complexity: Knots, Colourings, and Counting (London Math. Soc. Lecture Notes series 186), Cambridge University Press, Cambridge, 1993.

[Temperley(1972)] H. N. V. Temperley, in Combinatorics: Proc. Combinatorial Mathematics (D. J. A. Welsh and D. R. Woodall, Eds.). The Institute of Mathematics and its Applications, Oxford, 1972, pp. 356-357.

[Wu(1977)] F.-Y. Wu, Number of spanning trees on a lattice. J. Phys. A: Math. Gen. 10: L113-L115, 1977.

[Fortuin and Kasteleyn(1972)] C. M. Fortuin and P. W. Kasteleyn, On the random cluster model. I. Introduction and relation to other models. Physica 57: 536-564, 1972.

[Wu(1982)] F.-Y. Wu, The Potts model. Rev. Mod. Phys. 54: 235-268, 1982.

[Cori and Borgne(2003)] R. Cori and Y. Le Borgne, The sand-pile model and Tutte polynomials. Adv. Appl. Math. 30: 44-52, 2003.

[Dhar(2006)] D. Dhar, Theoretical studies of self-organized criticality. Physica A 369: 29-70, 2006. 
[Wu and Chao(2004)] B. Y. Wu and K.-M. Chao, Spanning Trees and Optimization Problems, Chapman \& Hall/CRC, Boca Raton, 2004.

[Temperley(1974)] H. N. V. Temperley, In Combinatorics (London Math. Soc. Lecture Note Series \#13) (T. P. McDonough and V. C. Mavron, Eds.). Cambridge University Press, Cambridge, 1974, pp. 202204.

[Chang and Shrock(2006)] S.-C. Chang and R. Shrock, Some exact results for spanning trees on lattices. J. Phys. A: Math. Gen. 39: 5653-5658, 2006.

[Chang and Wang(2006)] S.-C. Chang and W. Wang, Spanning trees on lattices and integral identities. J. Phys. A: Math. Gen. 39: 10263-10275, 2006.

[Chang(2009)] S.-C. Chang, Spanning trees on two-dimensional lattices with more than one type of vertex. J. Phys. A: Math. Theor. 42: 015208, 2009.

[Shrock and Wu(2000)] R. Shrock and F.-Y. Wu, Spanning trees on graphs and lattices in $d$ dimensions. J. Phys. A: Math. Gen. 33: 3881-3902, 2000.

[Tzeng and $\mathrm{Wu}(2000)]$ W.-J. Tzeng and F.-Y. Wu, Spanning trees on hypercubic lattices and nonorientable surfaces. Appl. Math. Lett. 13: 19-25, 2000.

[Aldous(1991)] D. Aldous, Asymptotic fringe distributions for general families of random trees. Ann. Appl. Probab. 1: 228-266, 1991.

[Manna et al.(1992)] S. S. Manna, D. Dhar and S. N. Majumdar, Spanning trees in two dimensions. Phys. Rev. A 46: R4471-R4474, 1992.

[Falconer(2003)] K. J. Falconer, Fractal Geometry: Mathematical Foundations and Applications. 2nd ed. Wiley, Chichester, 2003.

[Mandelbrot(1982)] B. B. Mandelbrot, The Fractal Geometry of Nature. Freeman, San Francisco, 1982.

[Alexander(1983)] S. Alexander, Superconductivity of networks. A percolation approach to the effects of disorder. Phys. Rev. B 27: 1541-1557, 1983.

[Daerden and Vanderzande(1998)] F. Daerden and C. Vanderzande, Sandpiles on a Sierpinski gasket. Physica A 256: 533-546, 1998.

[Dhar and Dhar(1997)] D. Dhar and A. Dhar, Distribution of sizes of erased loops for loop-erased random walks. Phys. Rev. E 55: R2093-R2096, 1997.

[Dhar(2005)] D. Dhar, Branched polymers on the given-Mandelbrot family of fractals. Phys. Rev. E 71: $031801,2005$.

[Domany et al.(1983)] E. Domany, S. Alexander, D. Bensimon and L. P. Kadanoff, Solutions to the Schrödinger equation on some fractal lattices. Phys. Rev. B 28: 3110-3123, 1983.

[Gefen et al.(1980)] Y. Gefen, B. B. Mandelbrot and A. Aharony, Critical phenomena on fractal lattices. Phys. Rev. Lett. 45: 855-858, 1980. 
[Gefen and Aharony(1981)] Y. Gefen and A. Aharony, Solvable fractal family, and its possible relation to the backbone at percolation. Phys. Rev. Lett. 47: 1771-1774, 1981.

[Gefen et al.(1983,1984)] Y. Gefen, A. Aharony and B. B. Mandelbrot, Phase transitions on fractals: I. Quasi-linear lattices. J. Phys. A: Math. Gen. 16: 1267-1278, 1983; Y. Gefen, A. Aharony, Y. Shapir and B. B. Mandelbrot, Phase transitions on fractals: II. Sierpinski gaskets, J. Phys. A: Math. Gen. 17: 435-444, 1984; Y. Gefen, A. Aharony and B. B. Mandelbrot, Phase transitions on fractals: III. Infinitely ramified lattices. J. Phys. A: Math. Gen. 17: 1277-1289, 1984.

[Guyer(1984)] R. A. Guyer, Diffusion on the Sierpinski gaskets: A random walker on a fractally structured object. Phys. Rev. A 29: 2751-2755, 1984.

[Hattori et al.(1990,1992)] K. Hattori, T. Hattori and S. Kusuoka, Self-avoiding paths on the preSierpinski gasket. Probab. Theory Relat. Fields 84: 1-26, 1990; T. Hattori and S. Kusuoka, The exponent for the mean square displacement of self-avoiding random walk on the Sierpinski gasket, Probab. Theory Relat. Fields 93: 273-284, 1992.

[Kozak and Balakrishnan(2002)(a)] J. J. Kozak and V. Balakrishnan, Analytic expression for the mean time to absorption for a random walker on the Sierpinski gasket. Phys. Rev. E 65: 021105, 2002.

[Kozak and Balakrishnan(2002)(b)] J. J. Kozak and V. Balakrishnan, Exact formula for the mean length of a random walk on the Sierpinski gasket. Int. J. Bifur. Chaos 12: 2379-2385, 2002.

[Rammal and Toulouse(1982)] R. Rammal and G. Toulouse, Spectrum of the Schrödinger equation on a self-similar structure. Phys. Rev. Lett. 49: 1194-1197, 1982.

[Chang and Chen (2008)(a)] S.-C. Chang and L.-C. Chen, Dimer-monomer model on the Sierpinski gasket. Physica A 387: 1551-1566, 2008.

[Chang and Chen (2008)(b)] S.-C. Chang and L.-C. Chen, Dimer coverings on the Sierpinski gasket. J. Stat. Phys. 131: 631-650, 2008.

[Chang and Chen (2008)(c)] S.-C. Chang and L.-C. Chen, Spanning forests on the Sierpinski gasket. Discret. Math. Theor. Comput. Sci. 10: 55-76, 2008.

[Chang and Chen (2009)] S.-C. Chang and L.-C. Chen, Number of connected spanning subgraphs on the Sierpinski gasket. Discret. Math. Theor. Comput. Sci. 11: 55-78, 2009.

[Chang et al.(2007)] S.-C. Chang, L.-C. Chen and W.-S. Yang, Spanning trees on the Sierpinski gasket. J. Stat. Phys. 126: 649-667, 2007.

[Harary(1969)] F. Harary, Graph Theory. Addison-Wesley, New York, 1969.

[Chang and Chen] S.-C. Chang and L.-C. Chen, Structure of spanning trees on the two-dimensional Sierpinski gasket. arXiv:0806.0721.

[Aldous(1990)] D. J. Aldous, The random walk construction of uniform spanning trees and uniform labelled trees. SIAM J. Disc. Math. 3: 450-465, 1990. 